\title{
Aldosterone deficiency in mice burdens respiration and accentuates diet-induced hyperinsulinemia and obesity
}

\author{
Wan-Hui Liao, ${ }^{1,2,3,4}$ Claudia Suendermann, ${ }^{2}$ Andrea Eva Steuer, ${ }^{5}$ Gustavo Pacheco Lopez, ${ }^{1,6}$ \\ Alex Odermatt, ${ }^{4,7}$ Nourdine Faresse, ${ }^{2,3,4}$ Maciej Henneberg, ${ }^{8,9}$ and Wolfgang Langhans ${ }^{1}$ \\ 'Physiology and Behavior Laboratory, Institute of Food, Nutrition and Health, ETH Zurich, Schwerzenbach, Switzerland. \\ ${ }^{2}$ Institute of Anatomy, University of Zurich, Zurich, Switzerland. ${ }^{3}$ Zurich Center for Integrative Human Physiology \\ (ZIHP), University of Zurich, Zurich, Switzerland. "National Center of Competence in Research "Kidney.CH", Switzerland. \\ ${ }^{5}$ Department of Forensic Pharmacology and Toxicology, Zurich Institute of Forensic Medicine, University of Zurich, Zurich, \\ Switzerland. ${ }^{6}$ Department of Health Sciences, Division of Biological and Health Sciences, Metropolitan Autonomous \\ University (UAM), Lerma, Mexico. 'Division of Molecular and Systems Toxicology, Department of Pharmaceutical Sciences, \\ University of Basel, Basel, Switzerland. ${ }^{8}$ Institute of Evolutionary Medicine, University of Zurich, Zurich, Switzerland. \\ ${ }^{9}$ Biological Anthropology and Comparative Anatomy Unit, University of Adelaide, Australia.
}

Aldosterone synthase inhibitors (ASIs) should alleviate obesity-related cardiovascular and renal problems resulting partly from aldosterone excess, but their clinical use may have limitations. To improve knowledge for the use of ASIs, we investigated physiology in aldosterone synthaseknockout (ASKO) mice. On regular chow diet (CD), ASKO mice ate more and weighed less than WT mice, largely because they hyperventilated to eliminate acid as $\mathrm{CO}_{2}$. Replacing $\mathrm{CD}$ with high-fat diet (HFD) lessened the respiratory burden in ASKO mice, as did 12- to 15-hour fasting. The latter eliminated the genotype differences in respiratory workload and energy expenditure (EE). Thus, aldosterone deficiency burdened the organism more when the animals ate carbohydrate-rich chow than when they ate a HFD. Chronic HFD exposure further promoted hyperinsulinemia in ASKO mice that contributed to visceral fat accumulation accompanied by reduced lipolysis, thermogenic reprogramming, and the absence of weight-gain-related EE increases. Intracerebroventricular aldosterone supplementation in ASKO mice attenuated the HFD-induced hyperinsulinemia, but did not affect $E E$, suggesting that the presence of aldosterone increased the body's energetic efficiency, thus counteracting the EE-increasing effect of low insulin. ASIs may therefore cause acid-overloadinduced respiratory burden and promote obesity. Their use in patients with preexisting renal and cardiopulmonary diseases might be contraindicated.

Conflict of interest: The authors have declared that no conflict of interest exists.

Submitted: February 1, 2018

Accepted: June 8, 2018

Published: July 25, 2018

Reference information: JCI Insight. 2018;3(14):e99015. https://doi.org/10.1172/jici. insight.99015.

\section{Introduction}

The emergence of the mineralocorticoid aldosterone supposedly promoted the evolution of terrestrial vertebrates (1). Aldosterone facilitates adaptation to land life by conserving salt and water in the intestine and kidney and facilitating renal acid elimination. Acid-base balance is crucial for all enzyme-based reactions. When metabolic rate and, hence, acid production increases, efficient acid elimination becomes essential, which in homeothermic mammals relies on lungs and kidneys. Aldosterone enhances renal acid elimination in the form of ammonium and through urinary acidification (2). Aldosterone deficiency therefore impairs renal acid excretion, and the ensuing metabolic acidosis elicits respiratory compensation.

Inappropriately high aldosterone levels, however, contribute to the development and progression of cardiovascular and renal diseases (3) and predispose for hypertension, diabetes, and hyperlipidemia (4). Consistent with its pathophysiological role, inhibiting aldosterone action with mineralocorticoid receptor (MR) antagonists or by blocking the renin-angiotensin system with angiotensin-converting enzyme inhibitors (ACEIs) or angiotensin-II receptor blockers (ARBs) slows kidney disease progression $(5,6)$. They also reduce cardiovascular mortality and improve health-related quality of life in heart failure patients $(7,8)$. However, aldosterone breakthrough, i.e., the return of aldosterone levels to or above pretreatment values, commonly reduces the efficacy of ACEIs and ARBs with chronic use (9). In addition, these medications do 
not protect against MR-independent actions of aldosterone on organ damage, such as aldosterone-induced pancreatic $\beta$ cell loss (10). These issues prompted the development of several potent aldosterone synthase inhibitors (ASIs) (11-14). Currently available ASIs improve some metabolic health parameters in preclinical models and clinical trials (14-16), but may not exclusively have beneficial effects. To improve basic knowledge for the safe use of ASIs, we used here the well-characterized aldosterone synthase-knockout (ASKO) mice (17) to investigate how low aldosterone levels affect physiology. As obesity is linked to hyperaldosteronism and a high risk of cardiovascular mortality (18), obese individuals supposedly benefit from ASI therapy. We therefore performed the experiments not only in mice fed regular chow diet (CD) but also in diet-induced obese (DIO) mice chronically fed a high-fat diet (HFD).

\section{Results}

ASKO mice fed $C D$ and maintained at $22^{\circ} \mathrm{C}$ overeat without increasing $B W$. CD-fed 2.5-month-old ASKO mice weighed less than WT controls (ASKO vs. WT: male, $23.1 \pm 1.4$ vs. $28.7 \pm 1.7 \mathrm{~g}$; female, $18.3 \pm 1.3$ vs. $22.5 \pm 2.0 \mathrm{~g}$ ) (Figure 1, A-C). Body composition, i.e., the weights of individual fat depots as well as the weights of brain, heart, kidney, spleen, and thymus were similar in ASKO and WT mice after normalization to BW (Figure 1D, and see below). Therefore, aldosterone deficiency restricts growth symmetrically and does not selectively affect the growth of soft tissue and organs or fat distribution. As reported previously (19), 5-month-old ASKO mice on CD were hyperkalemic, polyuric, and polydipsic with a lower BP compared with WT mice (Table 1 and Supplemental Figure 1; supplemental material available online with this article; https://doi.org/10.1172/jci.insight.99015DS1). Their tail arterial blood contained lower levels of bicarbonate (Supplemental Figure 2A) than those of WT mice and appeared slightly more acidic (ASKO, pH $7.31 \pm 0.01$; WT, pH $7.34 \pm 0.02$ ). Both genotypes had similar venous lactate levels (Supplemental Figure 2C).

ASKO mice ate more than WT mice relative to $\mathrm{BW}$ at $22^{\circ} \mathrm{C}$ (Figure 1E), despite aldosterone-deficiency-related metabolic acidosis (19), which in humans commonly reduces appetite (20). Their greater food intake is not a compensatory response to the reduced body $\mathrm{Na}^{+}$content. In both CD-fed and HFD-fed ASKO mice, salt supplementation ( $1 \% \mathrm{NaCl}$ drinking water; Supplemental Figure 3, G and P) increased — rather than decreased - food intake along with water intake (Supplemental Figure 3, A, C, J, and L). Moreover, the absolute amount of food consumed ad libitum was similar in both genotypes, as were the 1.5- and 3.5-hour intakes after 15-hour fasting (Figure 1, E and F). This suggests that aldosterone deficiency did not compromise the ability to adjust food intake in response to a change in body energy status. We then suspected a reduced intestinal uptake of short-chain fatty acids (SCFAs) in ASKO mice. SCFAs can provide $20 \%-30 \%$ of daily caloric requirements of omnivores, including mice (21). As aldosterone stimulates colonic $\mathrm{Na}^{+}$absorption (22) and increases enterocyte membrane fluidity (23), thus facilitating passive SCFA diffusion, aldosterone deficiency might impair $\mathrm{Na}^{+}$-coupled and -uncoupled SCFA transport in the large intestine (24). Surprisingly, ASKO mice lost less propionic and butyric acid in feces than WT mice (Figure 1G). Even after normalization to cecal SCFA contents, fecal SCFA levels in ASKO mice were still not higher than those in WT mice (Figure 1H). Daily fecal energy loss also did not differ between genotypes (Figure 1I). In sum, ASKO mice retain the capacity to regulate energy homeostasis in response to changes in body energy status. The increased food intake in ASKO mice is therefore unlikely an uncontrolled overeating resulting from aldosterone deficiency nor is it related to a reduced total body $\mathrm{Na}^{+}$content or to a reduced intestinal SCFA or nutrient absorption. The conversion of excess energy into fat was not affected by aldosterone deficiency either because in the nonfasting state plasma triglyceride (TG) and glucose levels were comparable in ASKO and WT mice, as was plasma insulin (Table 2).

Thermoneutral housing and fasting counteract the hyperventilation and increased energy expenditure of ASKO mice fed $C D$ at $22^{\circ} \mathrm{C}$. Food and fluid intake influence each other. The greater water intake in ASKO mice might therefore be related to overeating. Unsurprisingly, 16-hour water restriction drastically inhibited eating in both genotypes (Supplemental Figure 3, C and L). ASKO mice had a greater urinary $\mathrm{Na}^{+}$excretion/ $\mathrm{Na}^{+}$intake ratio than WT mice (Supplemental Figure 3, I and R), indicating they had a greater reduction in circulating volume. During water restriction, ASKO mice ate similar amounts of food and produced similar amounts of feces and urine as WT mice, but lost more weight (Supplemental Figure 3, A-E and J-N). This suggested greater energy expenditure (EE) in ASKO than in WT mice. Indirect calorimetry confirmed that ASKO mice maintained at $22^{\circ} \mathrm{C}$ had higher EE than WT mice, especially during the dark (active) and early light phase (Figure 2A) despite being as physically active as WT mice (Supplemental Figure 4C). ASKO mice were, however, hyperventilating, 

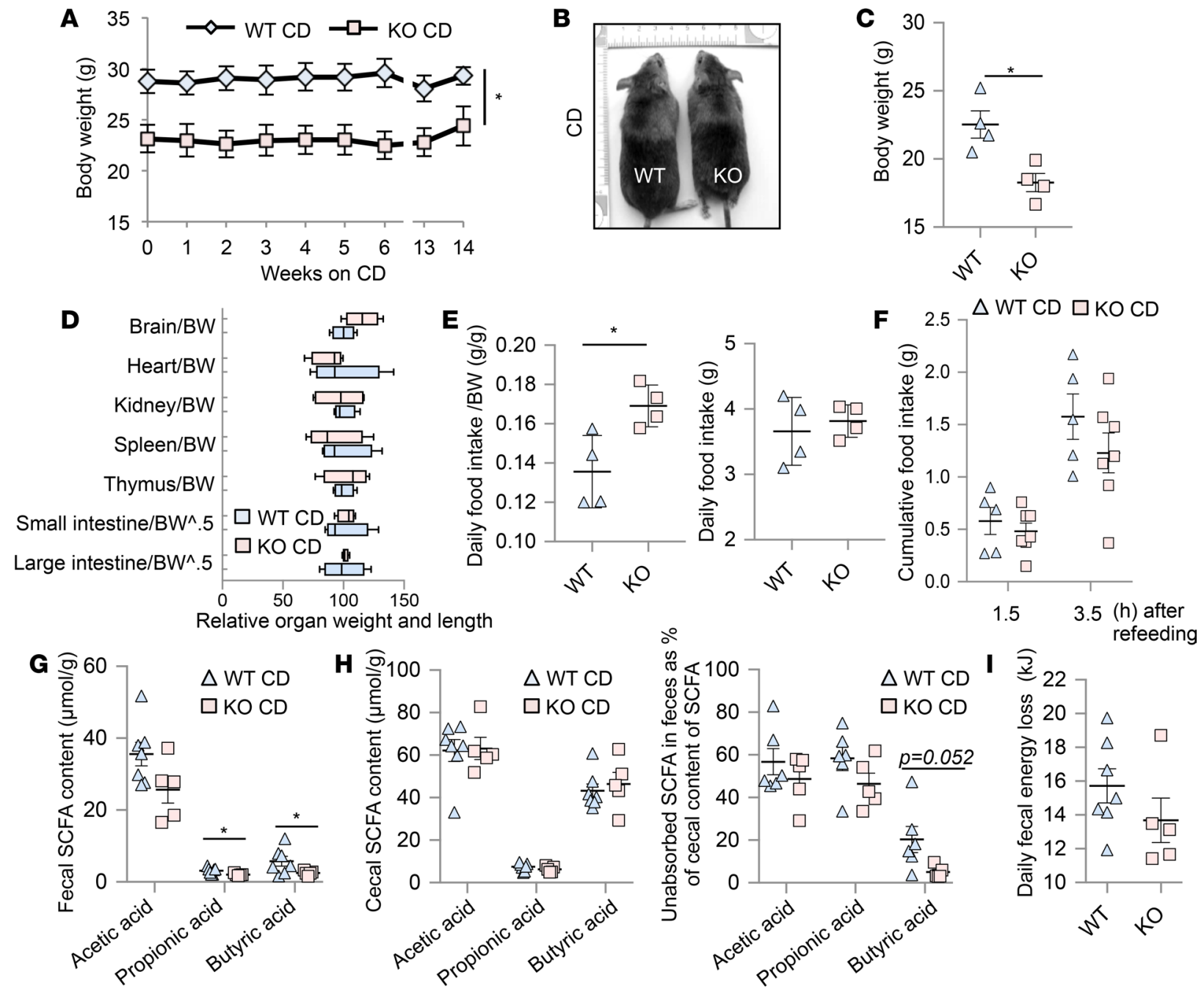

Figure 1. Chow diet-fed (CD-fed) ASKO mice at $22^{\circ} \mathrm{C}$ maintained BW at a lower level but ate more than WT mice, which was not related to decreased intestinal nutrient absorption. (A) BW tracked from 2.5 months of age ( $n=4 /$ group, mean \pm SD). (B) Adult WT and ASKO mice maintained on CD. (C) BW of 2.5-month-old female mice $(n=4 /$ group, mean \pm SD). (D) Box-and-whisker plot for relative organ size (organ mass/BW), small and large intestine length (organ length/BW ${ }^{0.5}$ ) of 2.5 -month-old female mice ( $n=4 /$ group). Box $=$ median + interquartile range, whisker $=10 \%-90 \%$ of distribution. WT mean set to 100. (E) Daily food intake of 6-month-old mice $(n=4 /$ group, mean \pm SD). (F) 1.5- and 3.5-hour food intake after 15 -hour fasting of 2.5-month-old mice ( $n=5-7 /$ group, mean \pm SEM). (G-I) Daily fecal short-chain fatty acid (SCFA) loss (G), cecal SCFA contents (left) and the ratio of fecal to cecal SCFA contents (right) $(\mathbf{H})$, and fecal energy loss of 6-month-old mice (I) ( $n=5$-7/group, mean \pm SEM). ${ }^{*} P<0.05$ WT versus ASKO by Mann-Whitney $U$ test (A, C, and $\mathbf{E})$ or by Welch's unpaired $t$ test $(\mathbf{F}-\mathbf{I})$.

as implied by a respiratory exchange ratio (RER) above 1 (Figure 2B) and by a trend toward a reduced $\mathrm{pCO}_{2}$ level in the tail arterial blood compared with WT mice (Supplemental Figure 2A). Their hyperventilation is presumably a compensation for reduced renal capacity to eliminate acids that results in less arterial bicarbonate to buffer acids compared with WT mice (Supplemental Figure 2A).

Hyperventilation is energetically costly; in humans the energy cost of breathing increases exponentially with enhanced ventilation (25). In the mid-dark phase, ASKO compared with WT mice displayed increases in both respiratory rate (RR) and minute volume (MV) (Figure 2, C and E), and had a shortened breathing cycle with a reduced expiratory time and a trend toward a reduced inspiratory time (Supplemental Figure 4, D and E). The high EE of ASKO mice may therefore be due to the extra work of breathing. Consistent with this possibility, the EE difference between the dark and light phases was greater in ASKO mice than in WT mice (Figure 2A). To further examine the role of hyperventilation in the high EE in CD-fed ASKO mice maintained at $22^{\circ} \mathrm{C}$, we alleviated their respiratory workload by $12-15$ hours of fasting at $22^{\circ} \mathrm{C}$ or by increasing the housing temperature to thermoneutrality. Fasting prompts cells to switch from burning carbohydrates to burning fat for fuel. Fat oxidation results 
Table 1. Water intake, urine osmolality, and urine and plasma electrolyte profiles of CD-fed WT and ASKO mice

\begin{tabular}{|c|c|c|}
\hline & \multicolumn{2}{|c|}{ CD } \\
\hline & WT & ASKKO \\
\hline Water intake (ml/20 g BW /24 h) & $2.82 \pm 0.20$ & $3.66 \pm 0.57^{A}$ \\
\hline \multicolumn{3}{|l|}{ Urine: } \\
\hline Volume (ml/20 g BW/24 h) & $0.48 \pm 0.06$ & $1.15 \pm 0.11^{A}$ \\
\hline Daily $\mathrm{Na}^{+}$excretion ( $\left.\mu \mathrm{mol} / 24 \mathrm{~h}\right)$ & $130.66 \pm 24.4$ & $195.23 \pm 21.28^{A}$ \\
\hline Urinary $\mathrm{Na}^{+}$excretion/ $\mathrm{Na}^{+}$intake (24 h) & $0.42 \pm 0.11$ & $0.59 \pm 0.03$ \\
\hline Daily $\mathrm{K}^{+}$excretion ( $\left.\mu \mathrm{mol} / 24 \mathrm{~h}\right)$ & $447.29 \pm 71.26$ & $547.85 \pm 72.82$ \\
\hline Urinary $\mathrm{K}^{+}$excretion/K+ intake (24 h) & $0.62 \pm 0.13$ & $0.71 \pm 0.07$ \\
\hline Creatinine clearance rate (ml/min) & $0.24 \pm 0.06$ & $0.32 \pm 0.04$ \\
\hline Osmolarity $\left(\mathrm{mOsm} / \mathrm{kg} \mathrm{H}_{2} \mathrm{O}\right)$ & $3,835 \pm 1,074$ & $1,780 \pm 289^{A}$ \\
\hline \multicolumn{3}{|l|}{ Plasma: } \\
\hline $\mathrm{Na}^{+}(\mathrm{mEq} / \mathrm{l})$ & $155.25 \pm 3.57$ & $156.05 \pm 3.89$ \\
\hline $\mathrm{K}^{+}(\mathrm{mEq} / \mathrm{l})$ & $4.14 \pm 0.24$ & $5.14 \pm 0.49^{A}$ \\
\hline
\end{tabular}

All values displayed as mean \pm SD. $n=4$ /group. ${ }^{A} P<0.05$ WT versus ASKO by Mann-Whitney $U$ test. in less $\mathrm{CO}_{2}$ production than carbohydrate oxidation for generating the same amount of ATP (26). It also reduces whole-body energy needs, which decreases $\mathrm{CO}_{2}$ production. Less $\mathrm{CO}_{2}$ production could alleviate the respiratory burden. Accordingly, fasting reduced RR, MV, and $\mathrm{EE}$ in both genotypes (Figure 2, C, E, and $\mathrm{F})$. It reduced $\mathrm{MV}$ and tidal volume more in ASKO than in WT mice (Figure 2, D and E) and eliminated the EE difference between genotypes (Figure $2 \mathrm{~F}$ ). These results suggest that at $22^{\circ} \mathrm{C}$ metabolizing carbohydrates imposes an energetic burden on the respiratory system and physiology of ASKO mice, which is alleviated by the prolonged fasting that enhances metabolizing fat.

The $22^{\circ} \mathrm{C}$ housing temperature represents a mild cold stress for mice. That increases $\mathrm{EE}$, body acid load, and hence ventilation by increasing metabolic rate $\left(\mathrm{CO}_{2}\right.$ production) (27) and activating brown adipose tissue (BAT). Accordingly, raising the housing temperature to $30^{\circ} \mathrm{C}$ (thermoneutrality) reduced $\mathrm{O}_{2}$ consumption and $\mathrm{CO}_{2}$ production and EE in both genotypes (Figure 2A and Supplemental Figure 4, A and B). The average RER of ASKO mice during the late $80 \%$ dark phase, however, was only mildly reduced, though never exceeded 1 throughout the light phase (Figure 2B), suggesting that ASKO mice still substantially relied on respiration to maintain acid-base balance at $30^{\circ} \mathrm{C}$. Such partial relief - as opposed to complete relief — of respiratory burden of ASKO mice by thermoneutral housing might explain why reductions in EE in ASKO mice were not significantly larger than those in WT mice in both dark and light phases (Figure 2A). Nonetheless, thermoneutral housing was sufficient to eliminate the genotype differences in $\mathrm{EE}, \mathrm{O}_{2}$ consumption, $\mathrm{CO}_{2}$ production, and food intake observed at $22^{\circ} \mathrm{C}$ (Figure $2 \mathrm{~A}$ and Supplemental Figure 4 , $\mathrm{A}, \mathrm{B}$, and F). Collectively, these data indicate that the genotype differences in EE and food intake at $22^{\circ} \mathrm{C}$ and on carbohydrate-rich CD are mainly due to hyperventilation in ASKO mice. The greater food intake in ASKO mice at $22^{\circ} \mathrm{C}$ is therefore likely a compensation for their higher EE, although it also contributes to the increased EE as would any physical activity.

ASKO mice are particularly prone to HFD-induced visceral adiposity and hyperinsulinemia. Fasting ASKO mice to lower body energy needs and to enhance fat utilization reduced the respiratory burden and EE to the levels of WT mice even under mild cold stress of $22^{\circ} \mathrm{C}$. We therefore studied how long-term HFD feeding affects energy balance in ASKO mice. We switched ASKO mice fed CD until the age of 2.5 months to HFD. After several weeks on HFD, ASKO mice gained more weight than WT. After 14 weeks on HFD, ASKO mice weighed more than WT mice $(42.7 \pm 1.7$ vs. $37.6 \pm 1.4 \mathrm{~g})$ and had more body fat (Figure 3, A-D), with no change in BP (Supplemental Figure 1). Perirenal, mesenteric, and BAT depots were enlarged (Figure 3E), and mesenteric adipocytes were larger in ASKO mice than in WT mice (7,075 \pm 194.6 and 4,929 $\pm 166.2 \mu \mathrm{m}^{2}$, respectively [mean $\pm \mathrm{SEM}$ ]) (Figure 3, F and G). mRNA analysis of Pparg and Cebpa, master regulators of adipogenesis, revealed no genotype differences (Figure $3 \mathrm{H}$ ). Collectively, these data indicate that aldosterone deficiency predisposes mice to HFD-induced visceral adiposity by enhancing adipocyte hypertrophy. Consistent with the morphological difference, ASKO mice had higher plasma insulin than WT mice after 14 weeks on the HFD (Table 2).

ASKO mice visceral adipose tissue shows reduced thermogenic reprogramming and lipolysis in response to chronic HFD feeding. Chronic HFD feeding produces diet-induced thermogenesis (DIT) in rodents. DIT partially manifests as an activation of the thermogenic program in BAT and white adipose tissue (WAT) (28). Analysis of BAT and perirenal WAT of 14-week HFD-fed mice by RT $^{2}$ profiler PCR array (Qiagen), focusing on adipose metabolism, revealed no differences between BAT of ASKO and WT mice. Perirenal WAT of ASKO mice, however, tended to have decreased expression of thermogenesis-related genes (Supplemental Figure 5A and Supplemental Table 4). Subsequent qRT-PCR revealed that chronic HFD feeding caused a 
Table 2. Plasma lipid profile and glucose and insulin levels in WT and ASKO mice maintained on CD or on HFD

\begin{tabular}{|c|c|c|c|c|c|}
\hline & & \multicolumn{2}{|c|}{ CD } & \multicolumn{2}{|c|}{ HFD } \\
\hline \multicolumn{6}{|c|}{ Metabolites and hormones } \\
\hline & Triglycerides (mg/dl) & $126.3 \pm 4.0$ & $123.0 \pm 29.4$ & $190.8 \pm 44.6$ & $126.0 \pm 53.7^{\mathrm{A}}$ \\
\hline & Free fatty acids (mM) & $0.53 \pm 0.17$ & $0.48 \pm 0.07$ & show & re $4 \mathrm{H}$ \\
\hline & Cholesterol (mg/dl) & $95.8 \pm 13.2$ & $129.8 \pm 29.8$ & $157.2 \pm 36.5$ & $148.0 \pm 30.3$ \\
\hline & Corticosterone (nM) & - & - & $408.7 \pm 97.4$ & $289.6 \pm 51.7$ \\
\hline & 11-Dehydrocorticosterone (nM) & - & - & $2.6 \pm 1.1$ & $3.6 \pm 0.7$ \\
\hline \multirow[t]{3}{*}{ Fasted } & Glucose (mg/dl) & - & - & $161.2 \pm 44.7$ & $138.7 \pm 29.5$ \\
\hline & Triglycerides (mg/dl) & - & - & $123.8 \pm 16.2$ & $72.1 \pm 12.8^{A}$ \\
\hline & Insulin (ng/ml) & - & - & $0.29 \pm 0.08$ & $0.25 \pm 0.05$ \\
\hline
\end{tabular}

All values displayed except insulin and aldosterone as mean $\pm S D, n=4-5$ /group in nonfasted condition, $n=3 /$ group in fasted condition. Values for insulin in both nonfasted and fasted condition and for aldosterone displayed as mean $\pm \mathrm{SEM}, n=3-7$ /group. ${ }^{\mathrm{A}} P<0.05$; ${ }^{\mathrm{B}} P<0.01$; ${ }^{\mathrm{C}} P<0.001$; HFD-fed WT versus HFD-fed ASKO or CD-fed WT versus CD-fed ASKO; Welch's unpaired $t$ test.

2- to 3-fold increase in the expression of core thermogenic genes, including uncoupling protein-1 (Ucp1), peroxisome proliferator-activated receptor $\alpha$ (Ppara), peroxisome proliferator-activated receptor $\gamma$ coactivator-1 $\alpha(P g c 1 a)$, and peroxisome proliferator-activated receptor $\gamma$ coactivator-1 $\beta(P g c 1 b)$, in perirenal WAT of WT mice (Figure 4A). This response was absent in ASKO mice (Figure 4A). Accordingly, their downstream targets, such as fatty acid oxidation enzymes and transcription factor-A, mitochondria (Tfam), nuclear respiratory factor-1 (Nrf1), and nuclear respiratory factor-2 (Nrf2) - principal transcription factors governing mitochondrial biogenesis - were expressed at reduced levels in the perirenal WAT of ASKO mice (Figure 4, B and C). Despite these changes, ASKO mice retain similar levels of mitochondrial mass and mitochondrial aerobic capacity to those of WT mice, as citrate synthase activity assay of perirenal fat (a surrogate of these 2 mitochondrial parameters) $(29,30)$ did not reveal genotype differences (Figure 4D). The absence of thermogenic reprogramming in perirenal fat of ASKO mice was paralleled by reduced lipolysis in their visceral adipose tissue (VAT). Compared with WT mice, ASKO mice expressed lower amounts of the active, phosphorylated form of hormone-sensitive lipase (HSL) in perirenal fat $(P=0.11)$ and mesenteric fat (Supplemental Figure 5D and Figure 4E). They also had reduced plasma nonesterified fatty acid (NEFA) levels and tended to have decreased liver TG content (Figure 4, F-H).

Thermogenic reprogramming and lipolysis in DIT are sympathetically stimulated with norepinephrine-activating $\beta 3$-adrenergic receptor ( $\beta 3$-AR). Assessing tyrosine hydroxylase $(\mathrm{TH})$, an indirect measure of sympathetic innervation (31), by immunofluorescence and immunoblotting revealed similar TH levels in VAT of both genotypes (Supplemental Figure 5, E and F). $\beta 3$-AR (Adrb3) in perirenal fat tended to be reduced only at the mRNA and not at the protein level (Supplemental Figure 5, G and H). These data suggest that the impaired lipolytic and thermogenic response of VAT, which makes HFD-fed ASKO mice more prone to developing central obesity, is unrelated to a reduced sympathetic input.

Switching from $C D$ to HFD feeding reduces the respiratory burden in ASKO mice. To elucidate the mechanisms underlying the increased susceptibility of ASKO mice to HFD-induced obesity at $22^{\circ} \mathrm{C}$, we focused on their changes in $\mathrm{EE}$ and food intake over time because aldosterone deficiency did not alter intestinal nutrient absorption (Figure 1I). After 10 weeks of HFD, the energy intake of both genotypes was reduced compared with 5-week HFD feeding (Figure 5D). Compared with CD consumption, HFD consumption increases the utilization of fat for fuel and, hence, decreases the production of $\mathrm{CO}_{2}$, which is the main stimulator of breathing. We therefore assumed that the switch to HFD would enhance metabolic efficiency in ASKO mice more than in WT mice because ASKO mice might then not have to hyperventilate much to achieve acid-base balance. Consistent with this assumption, 5-week HFD-fed ASKO mice eliminated less $\mathrm{CO}_{2}$ in the dark phase than those maintained on $\mathrm{CD}$ (Figure 5C). Also, while ASKO mice had greater CD-refeeding-induced increases in $\mathrm{EE}(35.10 \% \pm 5.50 \%$ in ASKO vs. $21.95 \% \pm 1.90 \%$ in WT mice), RR, 


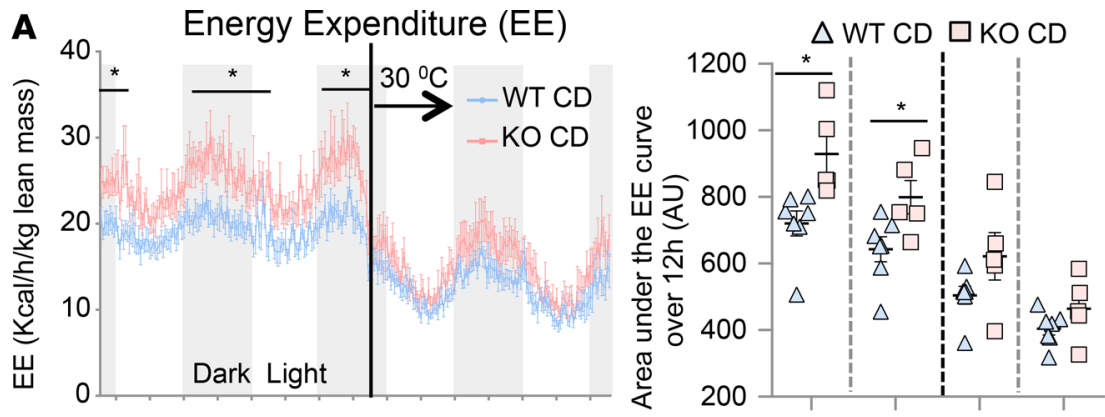

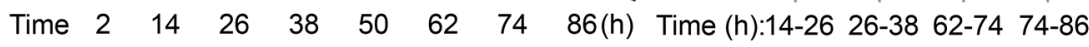
$22^{\circ} \mathrm{C}-\frac{62}{30^{\circ} \mathrm{C}}$

B Respiratory Exchange Ratio (RER)
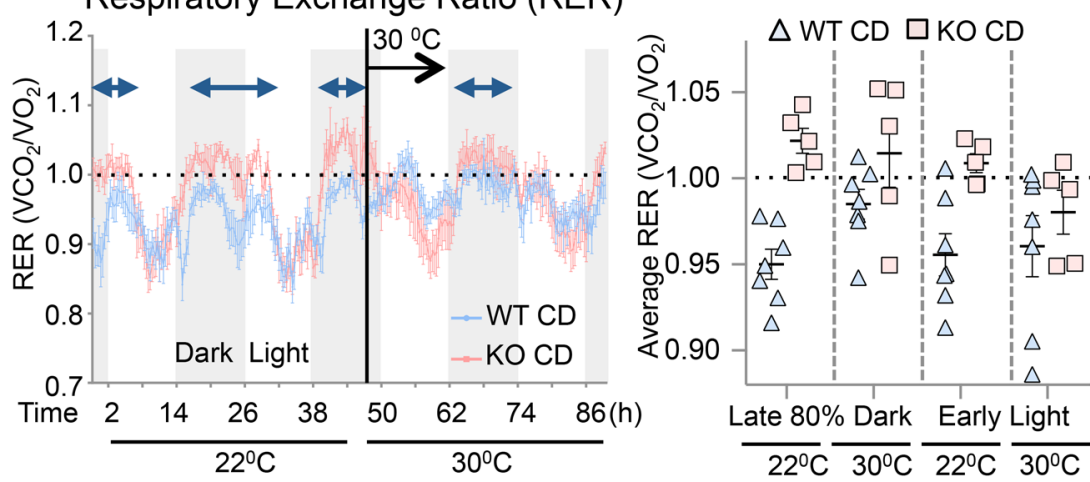
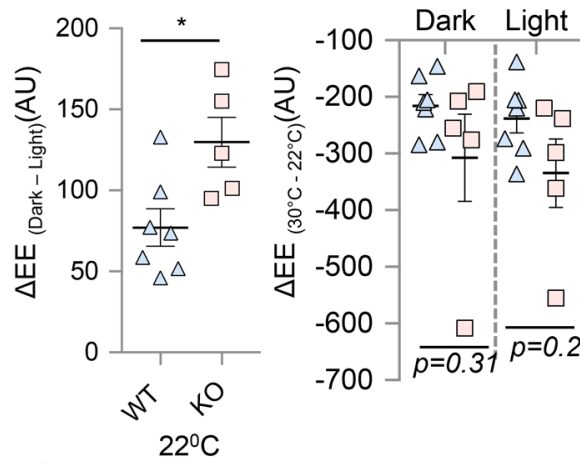

C
Respiratory Rate (RR) $\triangle$ WT CD $\square$ KO CD

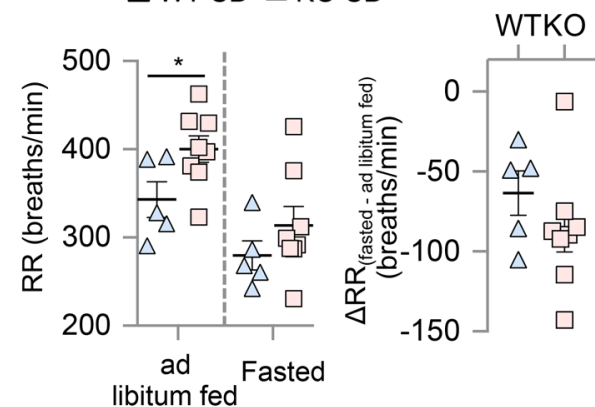

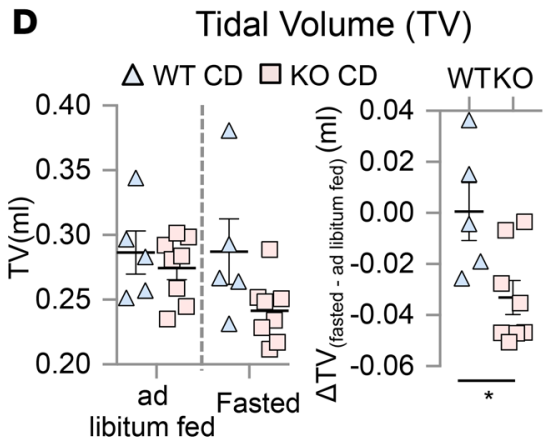

E

Minute Volume (MV)

\section{$\mathbf{F}$} $\triangle$ WT CD $\square$ KO CD WTKO
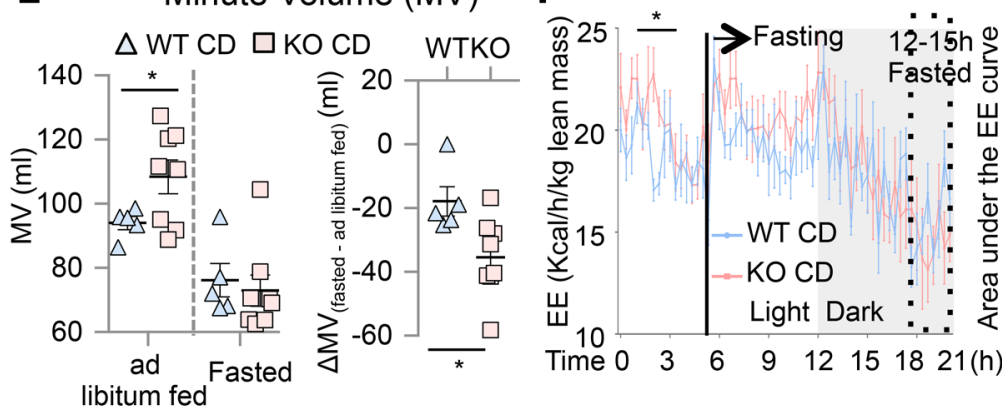

$\triangle W T C D$

$\square$ KO CD

Figure 2. Chow diet-fed (CD-fed) ASKO mice at $22^{\circ} \mathrm{C}$ were hyperventilating and had greater energy expenditure (EE) than WT mice in the dark and early light phase. Twelve to 15 hours of fasting eliminated and thermoneutral housing alleviated these changes. (A) Temporal changes in EE of 4-month-old mice at $22^{\circ} \mathrm{C}$ and $30^{\circ} \mathrm{C}$. Calculated changes in EE between dark and light phase at $22^{\circ} \mathrm{C}$ and between $22^{\circ} \mathrm{C}$ and $30^{\circ} \mathrm{C}$ housing of both genotypes ( $n=5-7 /$ group, mean \pm SEM). (B) Temporal changes in respiratory exchange ratio of 4-month-old mice at $22^{\circ} \mathrm{C}$ and $30^{\circ} \mathrm{C}(n=5-7 /$ group, mean \pm SEM). (C-E) Respiratory rate (RR) (C), tidal volume (TV) (D), and minute volume (MV) (E) of 3-month-old ad libitum-fed mice and after $12-15$ hours of fasting at $22^{\circ} \mathrm{C}$ and the calculated changes for each respiratory parameter between fasted and ad libitum fed ( $n=5$-8/group, mean \pm SEM). (F) Temporal changes in EE of 3-month-old mice at $22^{\circ} \mathrm{C}$ after food (CD) deprivation from the mid-light phase ( $n=5-7 /$ group, mean \pm SEM). ${ }^{*} P<0.05$ WT versus ASKO by Welch's unpaired $t$ test.

and MV than WT mice, they increased EE by only $20 \%$ in response to HFD refeeding, similar to WT mice (Figure 5E and Supplemental Figure 6A). WT and ASKO mice consumed similar amounts of food during the refeeding period of $\mathrm{CD}$ or of HFD (Supplemental Figure 6B). Refeeding ASKO mice with HFD increased respiratory workload less than refeeding them with $C D$. This clear differential response in EE and respiratory workload to different diets was absent in WT mice. This suggests that HFD feeding increased the susceptibility of ASKO mice to HFD-induced obesity by relieving the respiratory burden more than in WT mice.

Chronic HFD feeding increases EE $(32,33)$ through DIT and weight-gain-induced increases in cellular energy demand because some cells increase substantially in number (e.g., hematopoietic cells) (34-36) and some in both number and size (e.g., adipocytes). Cells obtain energy primarily by oxidizing nutrients, which generates $\mathrm{CO}_{2}$. Accordingly, WT mice steadily increased $\mathrm{EE}, \mathrm{O}_{2}$ consumption, and $\mathrm{CO}_{2}$ elimination with increasing weight (Figure 5, A-C). After 5 weeks on the HFD, EE of WT mice tended to be higher (7\%) than on CD, 


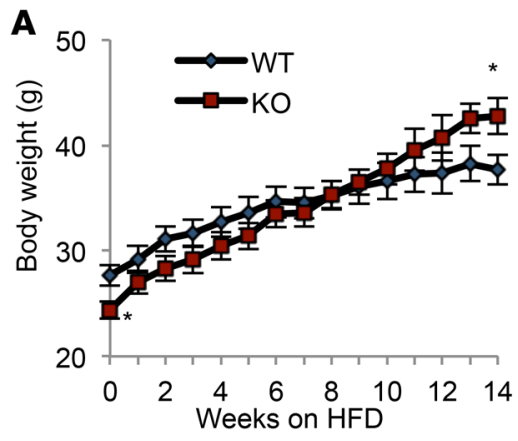

D

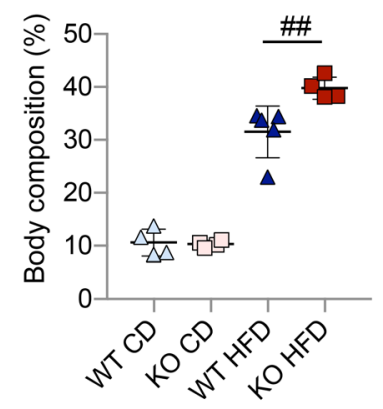

$\mathbf{F}$

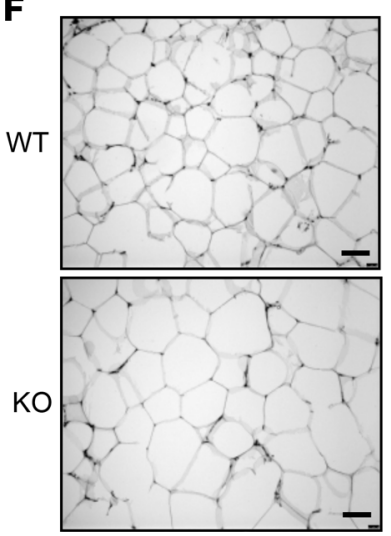

B

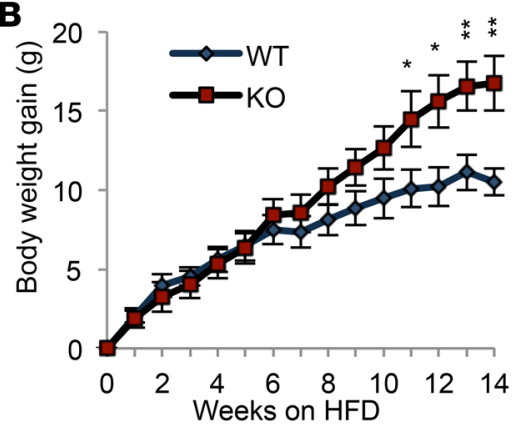

C

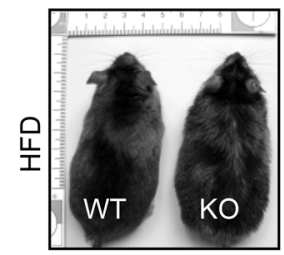

Lean

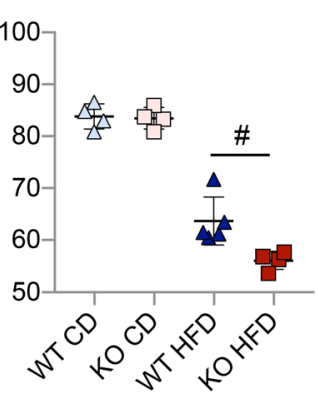

G

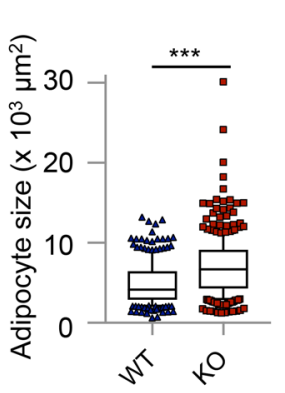

E

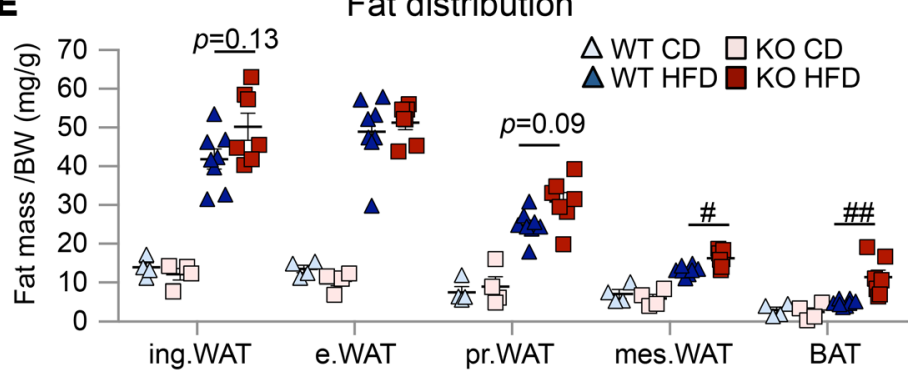

Figure 3. ASKO mice were more prone to high-fat diet-induced (HFD-induced) visceral adiposity. (A and B) BW and BW changes tracked from the age of 9 weeks ( $n=7-8 /$ group, mean \pm SEM). ${ }^{*} P<0.05,{ }^{* *} P<0.01$ HFD-fed WT versus HFD-fed ASKO by Welch's unpaired $t$ test. (C) WT and ASKO mice maintained on HFD for 12 weeks. (D and E) Body composition (D) and (E) weights of inguinal (ing.), epididymal (e.), perirenal (pr.) and mesenteric (mes.) white adipose tissue (WAT), and interscapular brown adipose tissue (BAT) after normalization to BW in mice fed chow diet (CD) and HFD ( $n=4-8 /$ group, mean $\pm \mathrm{SEM}$ ). ${ }^{*} P<0.05,{ }^{\# \#} P<0.01$ by 1 -way ANOVA followed by Tukey's post hoc test. (F and $\left.\mathbf{G}\right)$ Representative histological sections of H\&E-stained mesenteric fat of HFD-fed mice (F). Scale bars: $100 \mu \mathrm{m}$. (G) Box-and-whisker plot and distribution of adipocyte sizes of mesenteric fat ( $n=3$ [WT], $n=4$ [ASKO], 50-100 adipocytes/mouse). ${ }^{* *} P<0.001$ for mean adipocyte size difference between HFD-fed WT and ASKO mice by Welch's unpaired $t$ test. Box $=$ median + interquartile range, whisker $=10 \%-90 \%$ of distribution. (H) Pparg and Cebpa mRNA levels in perirenal fat ( $n=3 /$ group, mean \pm SD). Welch's unpaired $t$ test. Data in D-H were obtained from 2.5-month-old mice fed CD or HFD for another 14 weeks.

and after 5 more weeks this difference was significant (Figure 5A). Chronic HFD feeding also increased $\mathrm{O}_{2}$ consumption in both dark and light phases (10 weeks compared with 5 weeks, Figure 5B), and $\mathrm{CO}_{2}$ elimination appeared to be also increased, although the difference reached significance only in the light phase (Figure 5C). On the other hand, in ASKO mice chronic HFD feeding did not increase EE, and the genotype difference in EE observed at $22^{\circ} \mathrm{C}$ in mice fed the $\mathrm{CD}$ disappeared after 10 weeks on $\mathrm{HFD}$ (Figure 5A). $\mathrm{O}_{2}$ consumption and $\mathrm{CO}_{2}$ elimination in ASKO mice remained unchanged between 5 and 10 weeks of HFD feeding, although the animals became obese and had an activated thermogenic program in BAT (Supplemental Figure 7). Collectively, these results suggest that weight-gain-associated increases in EE were masked in HFD-fed ASKO mice. Though HFD feeding improves energetic efficiency in ASKO mice by alleviating the respiratory burden, this effect should have started early on after the switch from CD to HFD. We then proceeded to study whether chronic HFD feedinginduced hyperinsulinemia could influence adiposity progression in ASKO mice by affecting EE. 
A

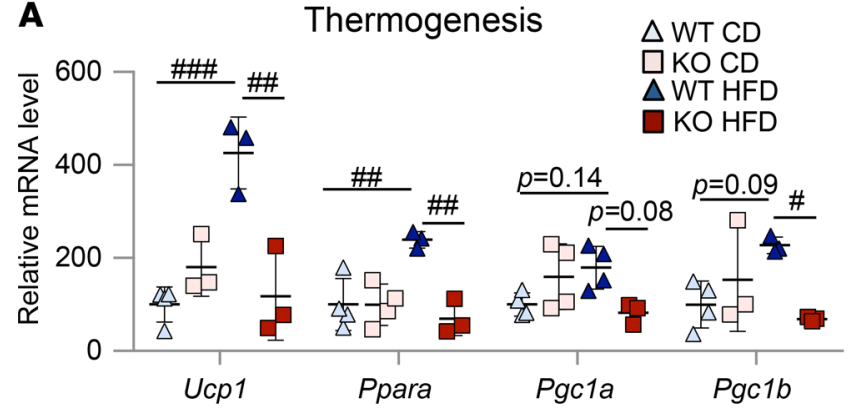

C $\triangle$ WT HFD $\square$ KO HFD

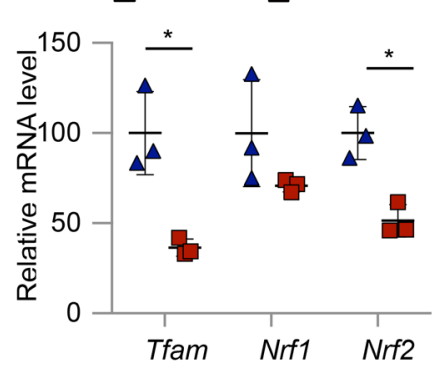

D

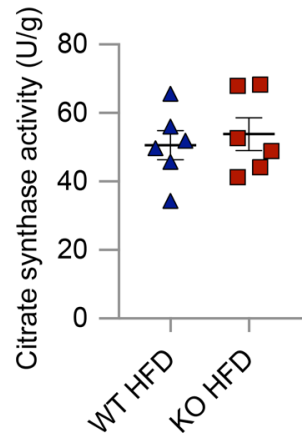

E

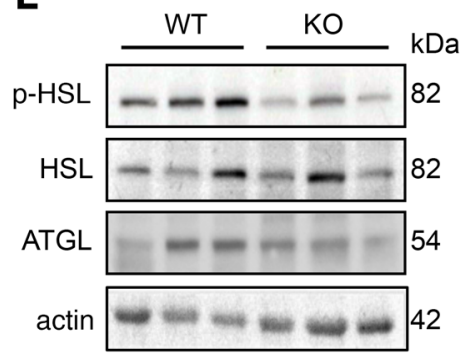

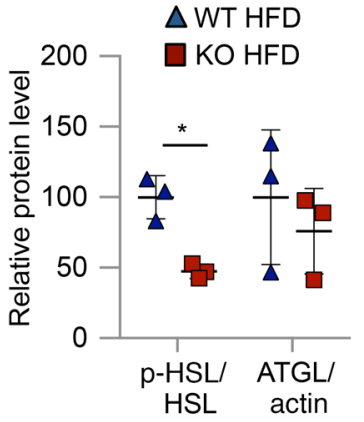

$\mathbf{F}$

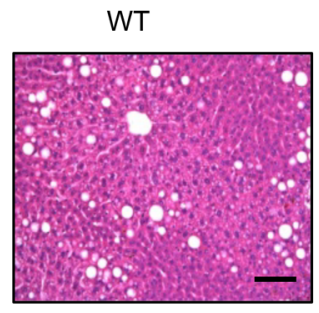

$\stackrel{*}{*} \underline{\underline{0} 0.21}$
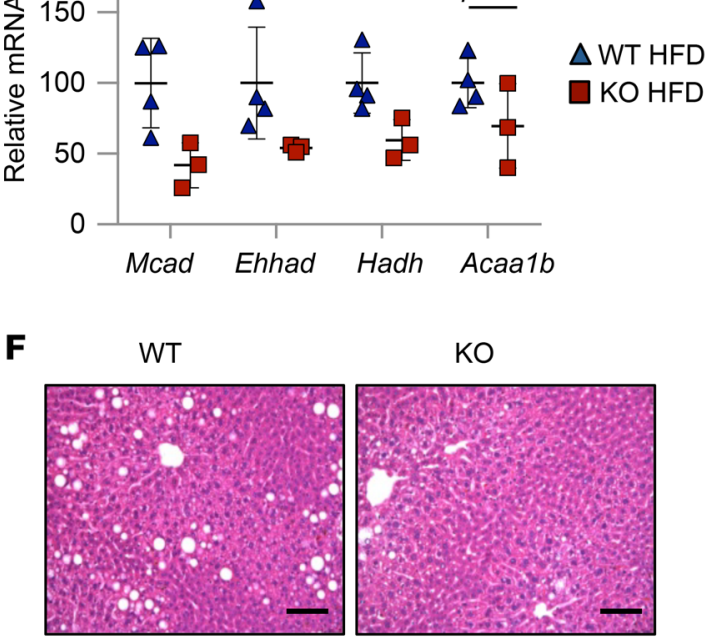

G

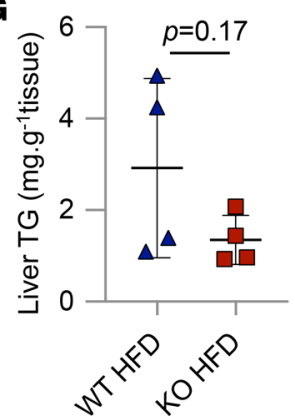

H

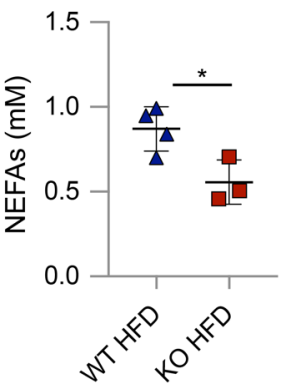

Figure 4. Visceral adipose tissue (VAT) of ASKO mice was unresponsive to 14-week high-fat diet (HFD) feeding-induced activation of the thermogenic program and lipolysis. (A) mRNA levels of thermogenic genes of perirenal fat of chow diet (CD) and HFD-fed WT and ASKO mice ( $n=3-4 /$ group, mean \pm SD). ${ }^{P}<0.05,{ }^{\#} P<0.01,{ }^{*} \# P<0.001$ by 1 -way ANOVA followed by Tukey's post hoc test. (B and $\mathbf{C}$ ) mRNA levels of enzymes involved in fatty acid oxidation (B) and in mitochondrial DNA transcription and replication in perirenal fat (C) of HFD-fed mice ( $n=3-4 /$ group, mean $\pm S D)$. ${ }^{*} P<0.05 \mathrm{HFD}$-fed WT versus HFD-fed ASKO by Welch's unpaired $t$ test. MCAD, medium-chain acyl-Coenzmye A dehydrogenase; EHHAD, enoyl-Coenzyme A hydratase; HADH, hydroxyacyl-Coenzyme A dehydrogenase; ACAA1b, acetyl-Coenzyme A acyltransferase 1b. (D) Citrate synthase activity in perirenal fat of 12- to 14-week HFD-fed mice ( $n=6$ /group, mean \pm SEM). Welch's unpaired $t$ test. (E) Western blot analysis of p-HSL, HSL, ATCL, and actin in mesenteric fat of HFD-fed WT and ASKO mice. Results presented after normalization to corresponding HSL and actin ( $n=3$ /group, mean \pm SD). ${ }^{*} P<0.05 \mathrm{HFD}$-fed WT versus HFDfed ASKO by Welch's unpaired $t$ test. HSL, hormone-sensitive lipase; ATCL, adipocyte triglyceride lipase. (F) Representative sections of H\&E-stained livers of HFD-fed WT and ASKO mice ( $n=4$ /group). Scale bars: $100 \mu \mathrm{m}$. (G and $\mathbf{H}$ ) Liver triglyceride (TC) contents (G) and plasma nonesterified fatty acid (NEFA) levels (H) of HFD-fed WT and ASKO mice ( $n=3$-4/group, mean \pm SD). ${ }^{*} P<0.05$ HFD-fed WT versus HFD-fed ASKO by Welch's unpaired $t$ test.

Chronic hyperinsulinemia contributes to the increased obesity in ASKO mice fed the HFD. Chronic HFD feeding increased adiposity and plasma insulin levels more in ASKO mice than in WT mice (Table 2). Hyperinsulinemia can be an accelerator of diet-induced obesity $(37,38)$ or an accompanying change resulting from the adiposity-dependent progression of insulin resistance (39). We therefore tracked plasma insulin in HFD-fed ASKO and WT mice over time. After only 1 week on the HFD, ASKO mice tended to have higher insulin levels than WT mice (Supplemental Figure 8C), and the difference reached significance by 8 weeks, when the percentage of fat in both genotypes was the same (Figure 6, A and B). This suggests that the higher insulin levels in ASKO mice are unlikely adiposity dependent. We further asked whether lowering the insulin levels of 14-week HFD-fed ASKO mice by overnight fasting (16 hours) would reactivate the suppressed lipolysis and transcriptional expression of thermogenic genes. As DIT does not cease with overnight fasting (40), there should still be a continuous sympathetic input to VAT under these conditions. 

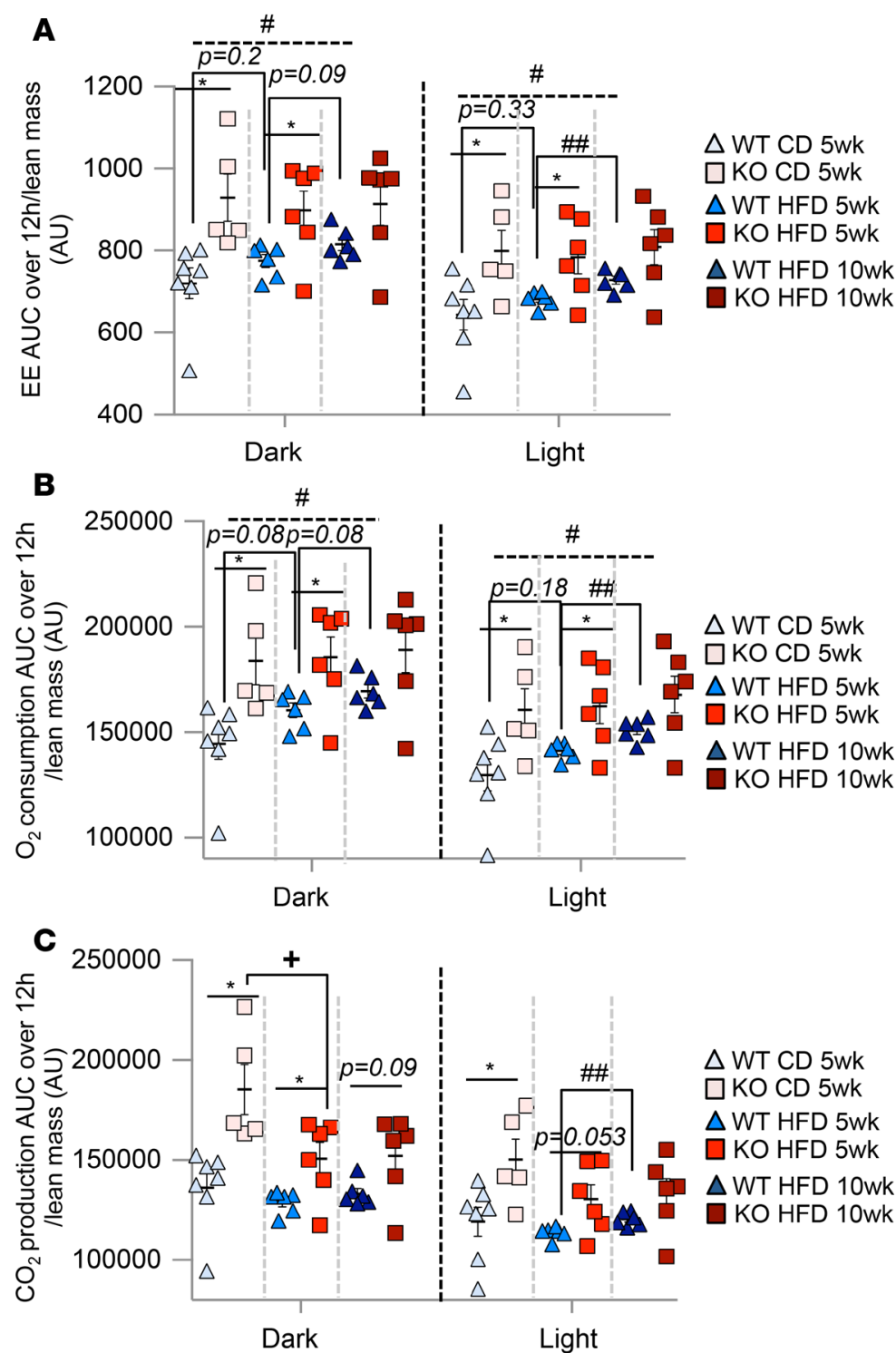

D $\triangle W T C D 5 w k \triangle W T H F D 5 w k \quad \triangle W T$ HFD 10wk $\triangle$ WT CD 5Wk $\square \mathrm{KO} C D 5 \mathrm{wk}$ $\triangle$ WT HFD 5 Wk $\square$ KO HFD 5wk $\triangle$ WT HFD 10wk $\square$ KO HFD 10wk

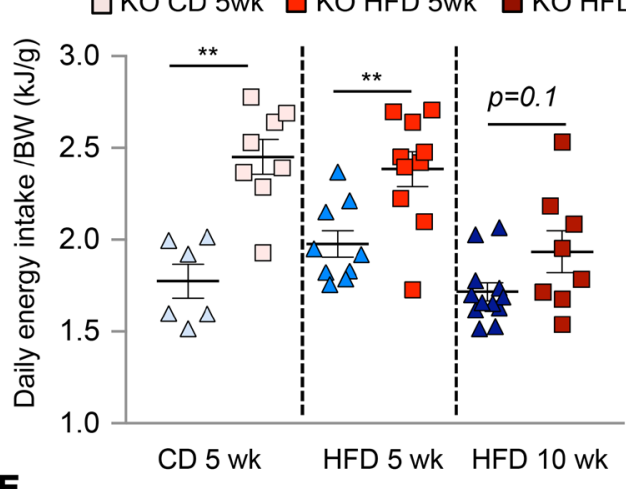

$\mathbf{E}$

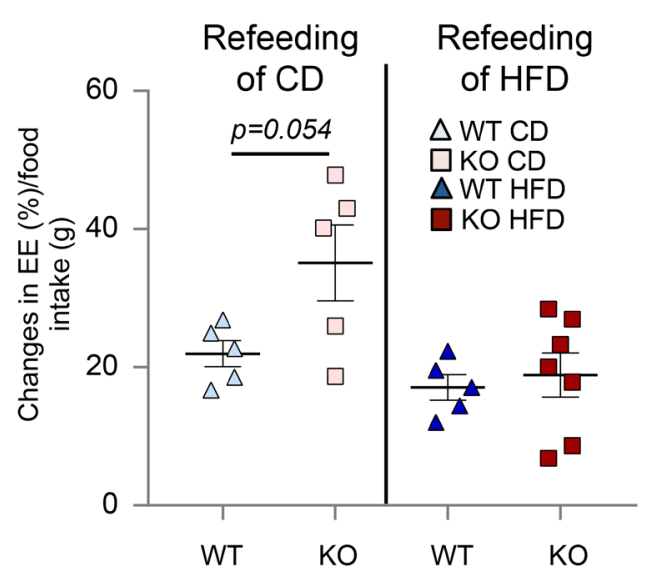

Figure 5. Switching from chow diet (CD) to high-fat diet (HFD) feeding reduced the respiratory burden in ASKO mice. Unlike in WT mice, chronic HFD feeding did not increase energy expenditure (EE) in ASKO mice. (A-C) AUC of (A) EE (B) $\mathrm{O}_{2}$ consumption (C) CO, production of CD-fed (5 weeks) and HFDfed (5 and 10 weeks) WT and ASKO mice ( $n=5$-7/group, mean \pm SEM). ${ }^{*} P<0.05$, CD-fed ASKO versus CD-fed WT or 5-week HFD-fed ASKO versus 5-week HFD-fed WT. ${ }^{~} P<0.05$, 10-week HFD-fed WT versus CD-fed WT. \#\# $P<0.01$, 10-week HFD-fed WT versus 5 week HFD-fed WT. ${ }^{\dagger} P<0.05$, CD-fed ASKO versus 5-week HFD-fed ASKO. (D) Daily energy intake ( $n=6-12$ /group, mean \pm SEM). ${ }^{* *} P<0.01$ CD-fed ASKO versus CD-fed WT or 5-week HFD-fed ASKO versus 5-week HFD-fed WT. (E) Changes in EE in response to refeeding of CD or HFD for 3.5 hours in 15-hour fasted, 3-month-old mice. Changes in EE = AUC EE following diet for 3.5 hours - AUC EE before diet for 3.5 hours. Fasting-refeeding was performed in the same mice, first with CD and 4 days later with HFD ( $n=5-7 /$ group, mean \pm SEM). All data analyzed by Welch's unpaired $t$ test. In A-C, data of CD-fed (5 weeks) mice are also shown in Figure $2 \mathrm{~A}$ and Supplemental Figure 4, A and B.

Overnight fasting reduced plasma insulin of WT and ASKO mice to a similar level (Table 2) and provoked VAT lipolysis in both genotypes similarly, as evidenced by their quantitatively similar increases in liver TG (Figure 6C). Consistent with reactivation of lipolysis, VAT of fasted ASKO mice showed an activation of the intracellular lipolytic cascade with p-HSL/HSL similar to that of WT mice and an even higher protein level of adipocyte triglyceride lipase (ATGL) (Figure 6E). Fasting also increased thermogenic gene transcripts similarly in WT and ASKO mice (Figure 6D). On the other hand, the homeostatic model assessment of insulin resistance suggested a similar degree of insulin resistance in WT and ASKO mice (Figure 6F). Collectively, these results suggest that the hyperinsulinemia is a cause rather than an effect of the increased susceptibility of ASKO mice to HFD-induced obesity. 
A

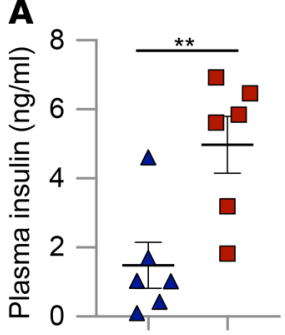

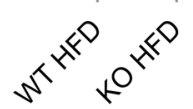

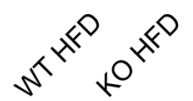

D

$\triangle$ ad libitum WT HFD $\square$ ad libitum KO HFD

$\triangle$ fasted WT HFD

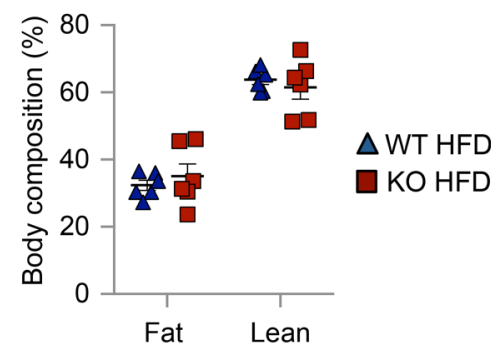

C

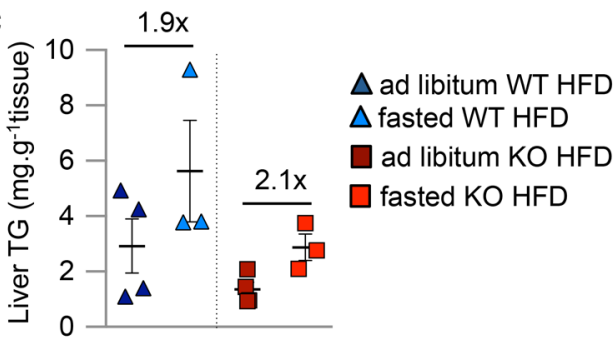

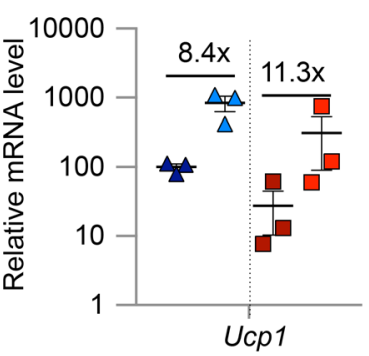
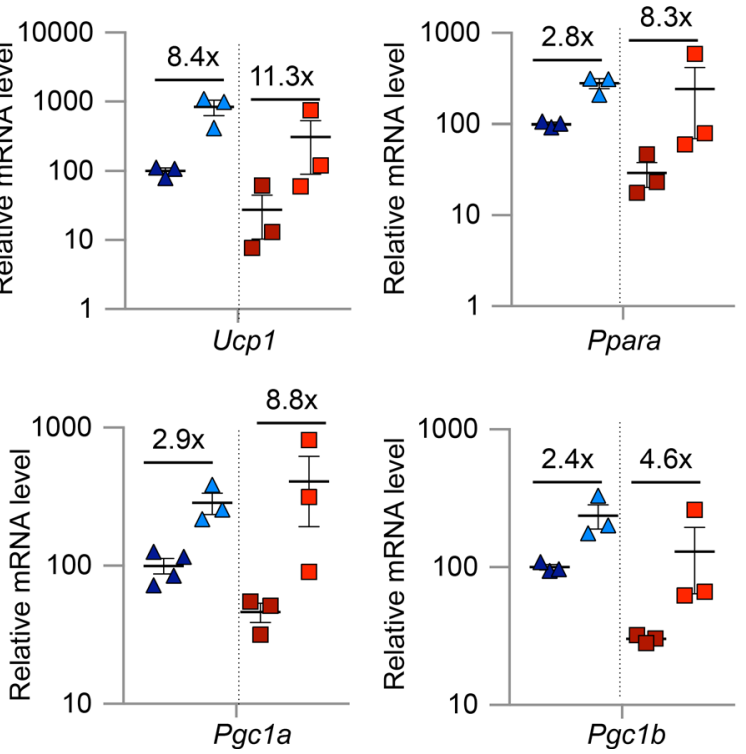
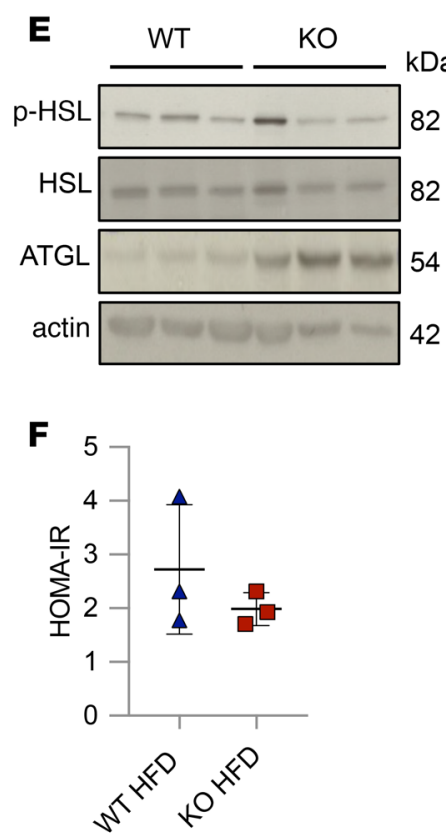

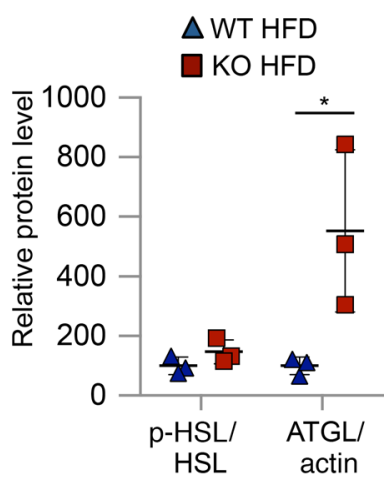

$\triangle$ WT HFD

$\square$ KO HFD

HSL actin

Figure 6. Visceral adipose tissue (VAT) of 14-week HFD-fed ASKO mice responded to 16-hour fasting-induced lipolysis and thermogenic gene activation. (A and B) Plasma insulin levels (blood collected from tail in the dark phase) (A) and body composition (B) of mice fed HFD for 8 weeks ( $n=6 /$ group, mean \pm SEM). (C) Liver triglyceride (TC) levels of HFD-fed WT and ASKO mice in nonfasted and fasted states ( $n=3-4 /$ group, mean \pm SD). (D) mRNA levels of thermogenic genes of perirenal fat of HFD-fed WT and ASKO mice $(n=3-4$ /group, mean \pm SD). (E) Western blot analysis of p-HSL, HSL, ATCL, and actin in mesenteric fat of fasted HFD-fed WT and ASKO mice. Results presented as relative protein levels of $p$-HSL and ATCL after normalization to corresponding HSL and actin ( $n$ $=3$ /group, mean \pm SD). (F) Insulin resistance determined using homeostatic model assessment (HOMA-IR) index. HOMA-IR was calculated by multiplying 16-hour fasting insulin ( $\mu \mathrm{U} / \mathrm{ml})$ with 16-hour fasting glucose (mmol/l) and dividing by 22.5 ( $n=3 /$ group, mean \pm SD). ${ }^{*} P<0.05,{ }^{* *} P<0.01$, HFD-fed ASKO versus HFD-fed WT by Welch's unpaired $t$ test (A, B, E, and F). In C and D, data of ad libitum HFD-fed mice are also shown in Figure 4, A and G.

I.c.v. infusion of aldosterone reduces plasma insulin and tends to attenuate weight gain in ASKO mice fed the HFD. HFD-induced hyperinsulinemia was lower in WT than in ASKO mice, but lack of endogenous peripheral aldosterone did not increase plasma insulin levels in CD-fed ASKO mice (Table 2). HFD feeding did not increase plasma aldosterone levels in WT mice (Table 2), suggesting that the central lack of aldosterone might be more important than peripheral aldosterone deficiency for regulating HFD-induced hyperinsulinemia. Adrenal-derived aldosterone appears to determine brain aldosterone levels (41). Chronic HFD feeding may have increased brain aldosterone in WT mice because it compromises the blood-brain barrier (42), potentially increasing the entry of aldosterone into the brain as occurs similarly for peripheral cholesterol (43). Because EE-reducing effects of hyperinsulinemia appear to be powerful enough to mask weight-gain-induced EE increases, whereas aldosterone deficiency causes an increase in energy turnover by respiration that is smaller with HFD than with $\mathrm{CD}$ feeding, aldosterone supplementation directly into the brain of HFD-fed ASKO mice may attenuate their hyperinsulinemia and weight gain.

Ten- to 12 -week HFD-fed ASKO mice were i.c.v. infused for 4 weeks at $25 \mathrm{ng} / \mathrm{h}$ with aldosterone dissolved in artificial cerebrospinal fluid (aCSF). I.c.v. aldosterone did not increase plasma aldosterone levels in WT mice, possibly because it suppressed endogenous aldosterone production after drainage 
into systemic circulation from cranial sinuses, but did raise plasma aldosterone levels of ASKO mice to almost two-thirds of those in WT mice (Figure 7B). I.c.v. aldosterone appeared to increase food intake in WT mice, likely because aldosterone can stimulate appetite (44). I.c.v. aldosterone tended to reduce weight gain and food intake and attenuated hyperinsulinemia in HFD-fed ASKO mice (Figure 7, A, C and D). As in WT mice, i.c.v. aldosterone did not increase $\mathrm{EE}, \mathrm{O}_{2}$ consumption, or $\mathrm{CO}_{2}$ production in ASKO mice (Figure 7, E-G), suggesting the reducing effects of aldosterone on EE through alleviating respiratory burden balanced the increasing effect of the reduced insulin level. Surprisingly, chronic aCSF infusion alone eliminated the difference in the level of pHSL/HSL between HFD-fed WT and ASKO mice. I.c.v. aldosterone decreased ATGL in HFD-fed ASKO mice, but had no effect in HFD-fed WT mice (Figure $7 \mathrm{H})$. Collectively, these results indicate that aldosterone has a suppressive effect on dietinduced hyperinsulinemia. The unchanged $\mathrm{EE}$ and gas exchange rates after i.c.v. aldosterone infusion in HFD-fed ASKO mice suggest that the attenuation of HFD-induced weight gain by i.c.v. aldosterone in ASKO mice is likely mediated by the reduction in food intake.

\section{Discussion}

Aldosterone facilitated the evolution of terrestrial vertebrates by enabling $\mathrm{Na}^{+}$and water conservation as well as renal acid excretion. Consistent with aldosterone's key role in electrolyte, hemodynamic, and acidbase homeostasis, CD-fed ASKO mice show renal salt wasting, hyperkalemia, low BP, low bicarbonate, and low BW throughout life (Table 1, Supplemental Figures 1 and 2, and Figure 1A), recapitulating human isolated aldosterone deficiency caused mainly by point mutations in the CYP11B2 gene $(45,46)$. Aldosterone deficiency also affects general growth in mice as it does in humans (Figure 1D and Figure 3D). Growth retardation in this scenario was not related to salt-wasting-induced extracellular fluid volume depletion but to aldosterone-deficiency-induced acidosis (47). Chronic metabolic acidosis impedes growth by inhibiting growth hormone (GH) secretion (48) and GH stimulation of insulin-like growth factor-1 release (49), and by reducing the sensitivity of the bone growth center to $\mathrm{GH}$ stimulation (50). A sustained alkaline therapy dose-dependently improves growth (51). It can be as effective as a therapy with fludrocortisone, an aldosterone mimetic (47), if the acid-base balance is restored (52). These clinical findings highlight the key role of aldosterone in acid-base balance regulation and the importance of maintaining an optimal $\mathrm{pH}$ for normal child growth. A positive energy balance is essential, but not sufficient, for growth success.

Adult, CD-fed ASKO mice at $22^{\circ} \mathrm{C}$ had greater EE than WT mice. The finding that ASKO mice had to spend extra energy to restore acid-base homeostasis by hyperventilation reiterates the importance of aldosterone in increasing energetic efficiency by facilitating renal acid excretion. Differences in thermoregulatory and cardiovascular adjustments due to cold stress may also contribute to the genotype differences in EE. Their contributions, however, are likely less than hyperventilation because the EE difference between dark and light phase was greater in ASKO mice than in WT mice, and because at each time point in the late light phase the EE difference appeared reduced. Also, fasting mice for $12-15$ hours at $22^{\circ} \mathrm{C}$ was sufficient to eliminate the genotype difference in EE (Figure 2, C-F). The extra energy costs of respiration might be particularly high during the active phase, accentuating the genotype difference in EE. With increasing ventilation, the energy cost of breathing increases exponentially because in addition to the more forceful contraction of primary respiratory muscles, accessory breathing muscles are recruited to stretch the thoracic wall $(53,54)$. Hyperventilation and increased EE in ASKO mice extended into the early light phase, suggesting that to wash out the acid buildup during dark takes time. Changes in EE were tied more closely to changes in ventilation in ASKO mice than in WT mice. Thermoneutral housing attenuated hyperventilation in ASKO mice, reducing the genotype difference in EE.

Chronic HFD feeding increased energetic efficiency in ASKO mice. By alleviating the respiratory burden and increasing insulin more than in WT mice, HFD feeding gradually increased BW more in ASKO than in WT mice. Using fasting-refeeding to minimize influences of the previous meal and of differences in energy status, we demonstrated that ASKO mice had reduced respiratory and EE responses to HFD compared with CD feeding. This reduction was not present in WT mice. Because ASKO mice relied more than WT mice on respiration to maintain acid-base balance, the relief of this respiratory burden induced by the CD-HFD switch saved more energy in ASKO than in WT mice. This contributed to the increased susceptibility of ASKO mice to HFD-induced obesity. ASKO mice on CD are like chronic obstructive pulmonary disease (COPD) patients, expending large amounts of energy for ventilation to maintain acid-base homeostasis; analogous to the present findings, a HFD nutritional supplement 
B

A

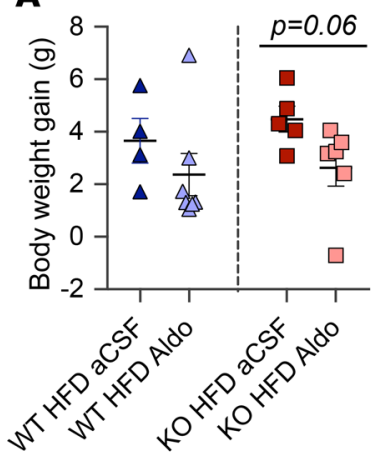

D

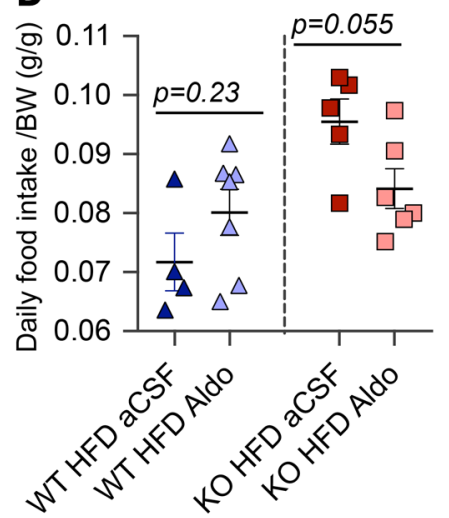

G

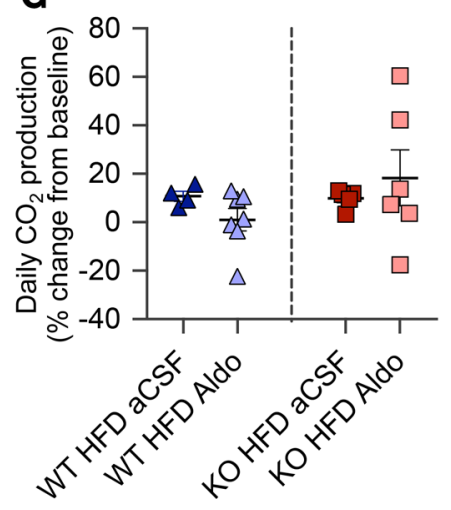

$\triangle$ WT HFD aCSF $\triangle$ WT HFD Aldo
C

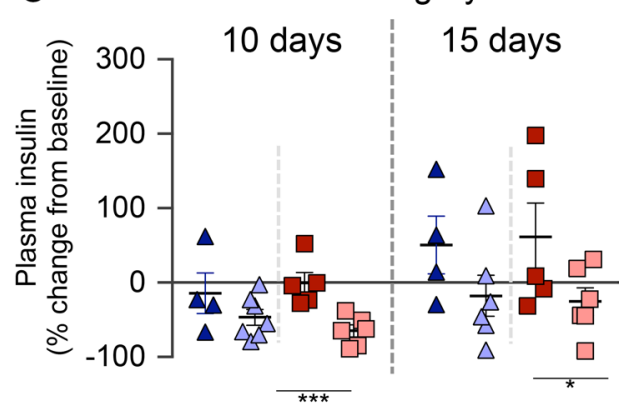

F

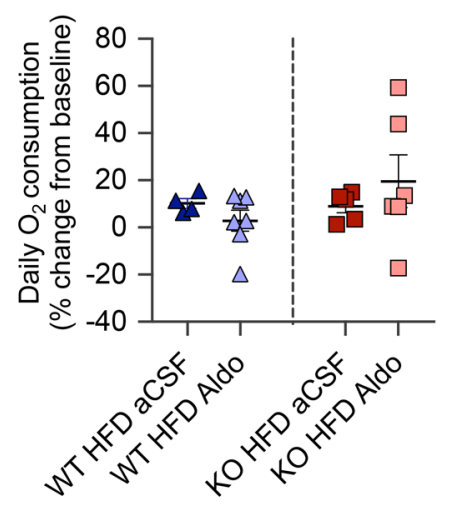

Post-surgery
E

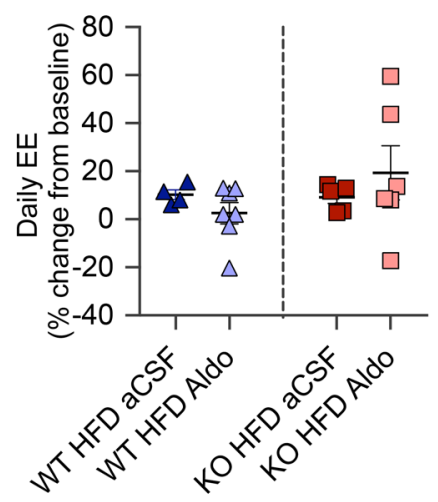

KO HFD aCSF $\square$ KO HFD Aldo
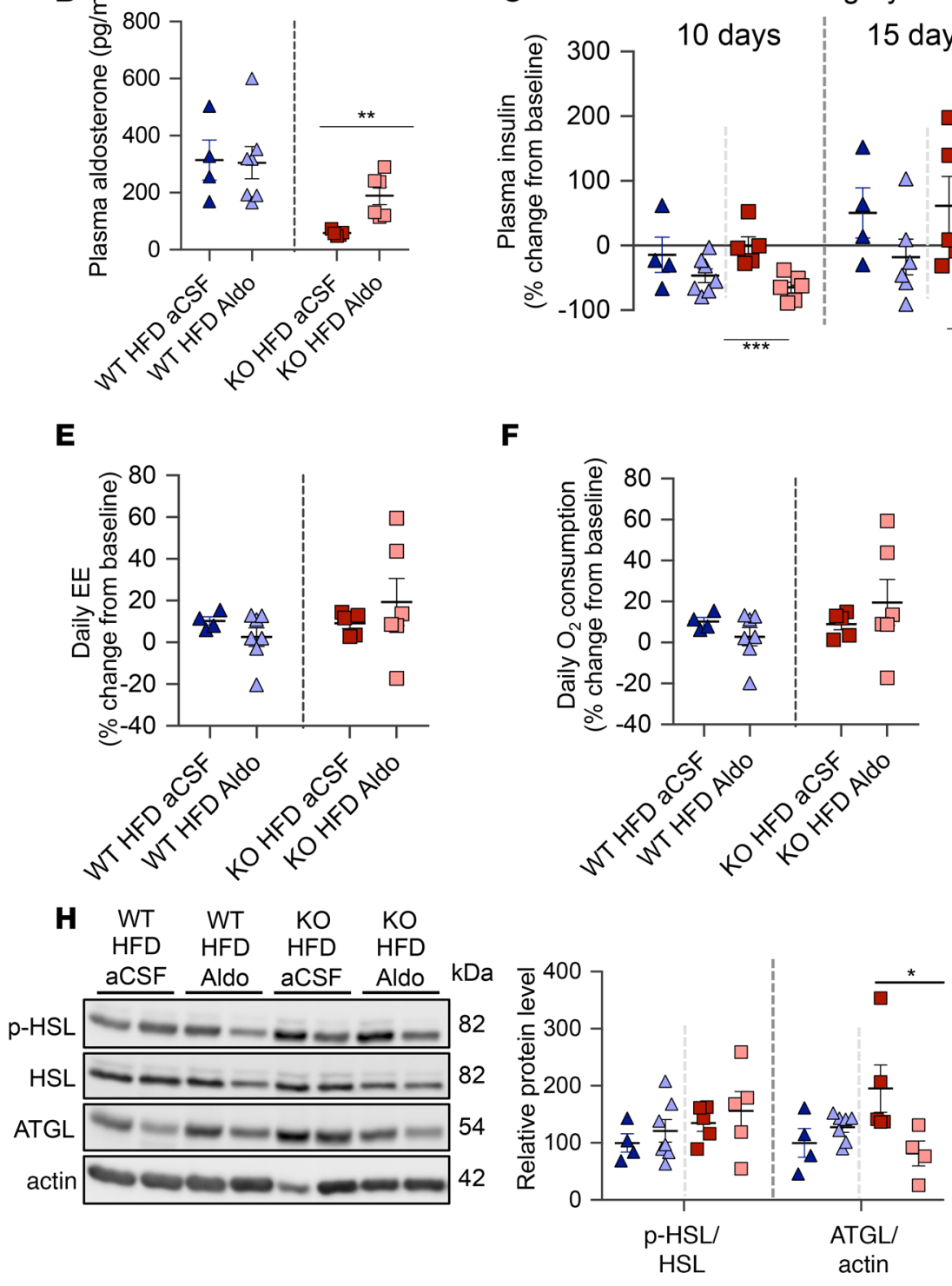

Figure 7. I.c.v. aldosterone supplementation attenuated hyperinsulinemia and tended to reduce BW gain in 10-12-week HFD-fed ASKO mice. (A) BW gain after 4-week i.c.v. infusion of aCSF or aldosterone ( $n=4-7 /$ group, mean \pm SEM). (B) Plasma aldosterone levels measured 4 weeks after infusion onset $(n=4-7 /$ group, mean \pm SEM). (C) Changes in plasma insulin levels 10 days and 15 days after infusion. Blood was collected from the tail at the dark phase $(n=4-7 /$ group, mean \pm SEM). (D) Daily food intake at 3-4 weeks after infusion ( $n=4-7 / g r o u p, ~ m e a n ~ \pm S E M)$. (E-C) Changes in daily (E) energy expenditure (EE), (F) $\mathrm{O}_{2}$ consumption, and (C) $\mathrm{CO}_{2}$ production measured 3-4 weeks after infusion ( $n=4-7 /$ group, mean $\pm \mathrm{SEM}$ ). (H) Western blot analysis of p-HSL, HSL, ATCL, and actin in mesenteric fat of HFD-fed WT and ASKO mice after 4-week i.c.V infusion of either aCSF or aldosterone. Results presented as relative protein levels of $\mathrm{p}-\mathrm{HSL}$ and ATCL after normalization to corresponding HSL and actin $\left(n=4-7 /\right.$ group, mean \pm SEM). ${ }^{*} P<0.05$, ${ }^{* *} P<0.01,{ }^{* * *} P<$ 0.001 ; aldosterone-infused ASKO versus aCSF-infused ASKO by Welch's unpaired $t$ test. aCSF, artificial cerebrospinal fluid.

in these patients reportedly alleviates the respiratory burden and minimizes the COPD-related weight loss (55-59). Aldosterone deficiency and COPD enhance diet-dependent effects on body acid load and thus, on ventilation. They indicate that a reduced body acid load might contribute to the often-described beneficial effects of low-carbohydrate/HFDs (60) and fasting/caloric restriction (61) on cardiopulmonary and vascular health (Figure 8), which has translational significance. Reducing $\mathrm{CO}_{2}$ generation by prompting cells to oxidize fat instead of carbohydrates and by reducing nutrient intake and consequent cellular metabolism should alleviate not only the pulmonary but also the cardiovascular burden because 


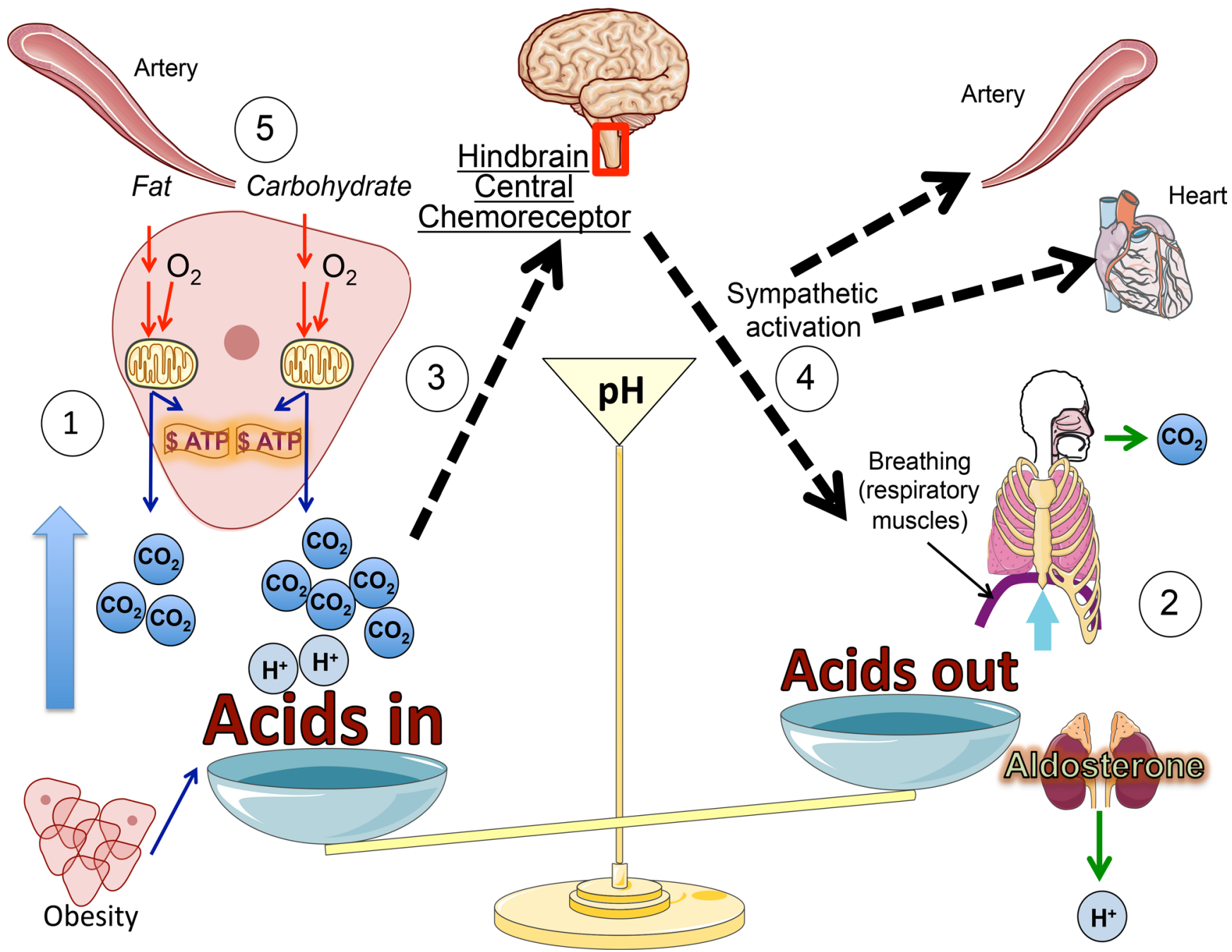

Figure 8. Potential beneficial effects of energy intake restriction or low carbohydrate intake (compared with isocaloric high carbohydrate intake) on the respiratory and cardiovascular systems. 1. Cells oxidize food-derived nutrients delivered by the arterial blood together with oxygen. Cellular respiration generates $\mathrm{CO}_{2}$ and other acidic metabolic waste (lactic acid, ketone bodies). 2. Lungs and kidneys cooperate to eliminate $\mathrm{CO}_{2}$ (lungs) and acids (kidneys). 3. Respiratory elimination of $\mathrm{CO}_{2}$ is under the control of the breathing center in the brainstem as part of a central chemoreceptor reflex. 4. Activation of central chemoreceptors stimulates breathing and enhances sympathetic output to the cardiovascular system, in essence increasing BP. 5. Carbohydrate oxidation generates more $\mathrm{CO}_{2}$ than fat (fatty acid) oxidation. Therefore, in conditions with reduced lung function (e.g., chronic obstructive pulmonary disease patients) and reduced renal acid elimination (e.g., aldosterone deficient mice), and/or hypermetabolism (e.g., obese people), promoting fat oxidation (fasting, isocaloric increase in fat intake at the expense of carbohydrates) might improve cardiovascular and pulmonary function.

it diminishes the central chemoreceptor reflex, which has implications in the progression of chronic heart failure (62). In this reflex, chemoreceptor activation in response to increased arterial $\mathrm{pCO}_{2}$, and indirectly to low $\mathrm{pH}$, stimulates the brainstem to enhance breathing and the sympathetic output to the vascular bed and heart. Therefore, such dietary intervention may especially benefit hypermetabolic patients such as viscerally obese people, who also happen to have an increased central chemoreflex sensitivity (63) and an enlarged VAT hampering respiration by impeding the contraction of the diaphragm.

In addition to the genotype differences in the energy-sparing response to the CD-HFD switch, ASKO and WT mice also differed in their hyperinsulinemic response to chronic HFD feeding. ASKO mice on HFD developed hyperinsulinemia sooner and more robustly than WT mice (Supplemental Figure 8), with insulin reaching nearly 5 times the levels of WT controls after 8 weeks on HFD, and were not more insulin resistant (Figure 6F). As chronic i.c.v. infusion of aldosterone in ASKO mice reestablished two-thirds of control plasma aldosterone levels and attenuated HFD-induced hyperinsulinemia (Figure 7, B and C), aldosterone deficiency obviously increases plasma insulin levels with HFD feeding. CD-fed ASKO mice also demonstrated a greater insulin response during a hyperglycemic clamp study than controls while being 
similarly insulin sensitive (10). Zucker diabetic fatty rats chronically on ASIs in an oral glucose tolerance test did the same (14). These findings suggest that a genetically or pharmacologically reduced aldosterone level somehow augments nutrient-dependent insulin release.

Hyperinsulinemia increases metabolic efficiency. Chronic insulin administration via osmotic minipumps reduced EE and increased BW in mice (37), and the prevention of chronic hyperinsulinemia in HFD-fed mice by a transgenic approach protected the animals from DIO (38). The elevated EE in the latter case was attributed to the WAT gaining BAT-like features, without changes in BAT. Similarly, the hyperinsulinemia in HFD-fed ASKO mice reduced thermogenic reprogramming and lipolysis only in WAT, but not BAT (Figure 4 and Supplemental Figures 5 and 7). Decreased levels of UCP1 enhance oxidative phosphorylation and slow down temperature-dependent biochemical reactions, thus minimizing the energy loss from energy transformation and futile cycles. Insulin, by limiting gluconeogenesis and lipolysis and promoting nutrient storage (64), also reduces the nutrient utilization rates and thus also the respiratory workload and $\mathrm{EE}$ because less $\mathrm{O}_{2}$ is necessary and less $\mathrm{CO}_{2}$ must be eliminated.

Chronic i.c.v. infusion of aldosterone attenuated diet-induced hyperinsulinemia. Yet, the reduced insulin levels did not accordingly increase EE in HFD-fed ASKO mice (Figure 7, C and E), indicating that the presence of aldosterone in ASKO mice counteracts the influence of hyperinsulinemia on energy efficiency. Because the gas exchange rate of HFD-fed ASKO mice also remained unchanged following aldosterone supplementation (Figure 7, E-G), the aldosterone presence in HFD-fed ASKO mice may reduce ventilation-related energy cost and, hence, offset the increase in EE resulting from reduced circulating insulin. Unfortunately, we could not assess whether aldosterone supplementation to HFD-fed ASKO mice reactivates lipid catabolism in the VAT because aCSF infusion alone activated lipolysis in these mice (Figure $7 \mathrm{H}$ ). Chronic aCSF infusion may influence VAT lipolysis by altering CSF ion concentrations, which affects sympathetic activities $(65,66)$. ASKO and WT mice presumably have different CSF compositions, because aldosterone regulates CSF ion concentrations (66), i.c.v. infusion of normal aCSF may therefore change CSF ionic profiles in ASKO mice. On the other hand, i.c.v. aldosterone infusion tended to attenuate HFD-induced BW gain in ASKO mice. This may be related to the parallel trend towards a reduction in food intake (Figure 7D), which could be due to central or peripheral effects of aldosterone or to changes in CSF electrolyte content in ASKO mice.

Previous studies exploring aldosterone's effects on adiposity, often with MR blockers, yielded inconsistent results $(67,68)$. This is perhaps due to varying antiandrogenic or progesterogenic activity of the MR blockers employed $(69,70)$, or because they differentially inhibited anabolic actions of glucocorticoids or MR-mediated adipogenesis (71). Moreover, MR blockers might shift energy balance by simply altering food intake or physiological activities, as MRs are widely expressed in epithelial tissues and metabolic organs. MRs in the hypothalamic paraventricular nucleus, in particular, are under the control of aldosterone and are involved in regulation of salt homeostasis and pressor responses to stress (72).

In sum, using the preclinical model of ASKO mice, we characterize aldosterone as an important hormone increasing energetic efficiency. Aldosterone evolved to conserve salt and fluid and to facilitate efficient acidic metabolic waste removal in terrestrial vertebrates. By minimizing acid-base disturbance and respiratory burden, aldosterone increases the organism's resilience to stress and diseases that are characterized by hypermetabolism. This may explain why during acidosis in mammals, aldosterone levels are increased (2). Hyperaldosteronism in human obesity may result from the increased body acid load due to enhanced cell respiration and respiratory workload. The recent concept of mitigating aldosterone's toxicity to limit organ failure is supported by several studies $(16,73)$, but the physiological importance of aldosterone in maintaining acid-base and electrolyte homeostasis as demonstrated here should not be neglected when considering reducing aldosterone level for treatment. By burdening acid-base balance and causing hyperkalemia, reduced aldosterone levels may not only unnecessarily complicate the medical conditions as demonstrated in a recent case report (74), but also endanger life particularly in patients with preexisting renal insufficiency or pulmonary diseases. Moreover, as the ventilation increase relies on the activation of the chemoreceptor reflex, which also stimulates sympathetic activity in the cardiovascular system and is involved in chronic heart failure (Figure 8), any presumed positive effect of ASIs on cardiovascular health may be counteracted. Aldosterone deficiency also accentuated HFD-induced hyperinsulinemia that promoted visceral fat expansion. Therefore, in subjects who prefer HFD, ASIs may increase visceral adiposity, which potentially further aggravates the cardiovascular and pulmonary burden in viscerally obese people. Though our findings require validation in human subjects, we recommend paying attention to potential negative effects on the cardiovascular and pulmonary system and on 
BW gain when launching ASI-related clinical studies. Based on our work and published studies (26, 32, 53, $55,57,59,60,62,63)$, we suggest to focus on strategies to reduce body acid load, such as dietary intervention to improve cardiovascular health (Figure 8).

\section{Methods}

Animal experiments. WT and ASKO mice (129SvEv background) were maintained at $22^{\circ} \mathrm{C}$ under a $12-\mathrm{h}$ light/dark cycle with free access to food and tap water. The ASKO mouse model, generated in the laboratory of Olivier Smithies, University of North Carolina, Chapel Hill, North Carolina, USA, was provided by Johannes Loffing, University of Zurich, Zurich, Switzerland. Unless otherwise indicated, only male mice were used and they were sacrificed in the nonfasted state.

Fourteen-week DIO study. Ten-week-old WT and ASKO mice were randomly assigned to either CD (Kliba 3436, Provimi Kliba AG) or HFD (D12492, Research Diets) (for diet composition see Supplemental Table 1) for 14 weeks. BW was assessed weekly. One HFD-fed ASKO mouse developed a skin infection in week 10 of HFD feeding and was excluded from the experiment since then.

After week 14, individual body composition was analyzed by NMR (EchoMRI-100, EchoMedical Systems). Data for each mouse represent the average of 2 consecutive measurements. One week later, mice were singly housed in metabolic cages (Tecniplast) to monitor daily water and food intake, fecal and urinary production, and to collect urine. The experiment comprised a 3-day adaptation and a 2-day measurement period. Another week later, all CD-fed and some HFD-fed mice were anesthetized (isoflurane, 2.5\% in $\mathrm{O}_{2}$ ) and sacrificed in the nonfasted state in the early to mid-light phase. The remaining HFD-fed mice were sacrificed after 16 hours of fasting. Immediately after thoracotomy, blood was collected from the right ventricle into EDTA-treated Eppendorf tubes followed by transcardial PBS perfusion (40 ml) and tissue collection for molecular (snap frozen in liquid nitrogen) and histological analysis.

$B P$ measurement. In week 10 of CD or HFD feeding, systolic, diastolic, and mean BP (SBP, DBP, and MAP, respectively) were measured (tail cuff method, model BP-2000, Visitech Systems) in the light phase for 3-5 consecutive days following 5 training days, as previously described (75). The SBP, DBP, and MAP of each mouse were determined as the average of measurements on days 3-5. Each day, measurement comprised 9-15 of 20 automatic inflation/deflation cycles in total. Five to 11 daily measurements were discarded as "systolic time-out," often due to excessive movement of the mouse. Finally, the results were averaged for all mice of each genotype.

Arterial and venous blood collection for blood gas and lactate analysis. Six- to 9-month-old male and female mice maintained on $\mathrm{CD}$ at $22^{\circ} \mathrm{C}$ were restrained using a mouse restrainer during tail artery blood collection. After immersing the tail in $37^{\circ} \mathrm{C}$ tap water for $10-15$ seconds, the ventral tail artery was nicked with a scalpel blade at one-third to one-fifth of tail length from the anus. About $120 \mu$ arterial blood was collected into a heparin-coated vial and immediately analyzed for $\mathrm{pH}, \mathrm{pO}_{2}$, and $\mathrm{pCO}_{2}$. Then, $150 \mu 1$ of venous blood was obtained by puncturing the superficial temporal vein for blood gas and for lactate analysis, the latter by a portable lactometer (StatStrip Lactate Xpress, Nova Biomedical). The bicarbonate content of the blood sample was automatically calculated by a blood gas analyzer (model ABL80 FLEX CO-OX, Radiometer) from the measured $\mathrm{pH}$ and $\mathrm{pCO}_{2}$ (Henderson-Hasselbalch equation).

Whole-body plethysmography. Respiratory parameters (RR, tidal volume, MV, inspiratory and expiratory time) of conscious, unrestrained mice were obtained during mid to late dark phase using whole-body plethysmography (WBP) (FinePointe; Buxco/DSI). WBP measures box flow ( $\mathrm{ml} / \mathrm{min}$ ) created by pressure changes inside the chamber resulting from mouse breathing. By applying Boyle's law to box flow, WBP derives estimates of mouse respiratory flow and volume. Mice were placed individually into the WBP chamber continuously supplied with room air at a rate of $11 / \mathrm{min}$. All mice were habituated to the chamber for 40 minutes each day for 2 days prior to the measurements ( 25 minutes preceded by 15 -minute acclimation). Results for ad libitum-fed mice represent the average of 3 consecutive day measurements. Results for mice in fasted and refed status were obtained after 12-15 hours of fasting and 1.5 hours of refeeding.

Indirect calorimetry and locomotor activity. Singly caged mice fed CD or HFD for different periods were placed into the individual metabolic chambers with free access to food and water for 4-6 days: 2 days adaptation followed by 2 days for the measurement at $22^{\circ} \mathrm{C}$ and, in some experiments, another 2 days at $30^{\circ} \mathrm{C}$. $\mathrm{O}_{2}$ consumption, $\mathrm{CO}_{2}$, RER, EE, and locomotor activity were analyzed by a PhenoMaster system (TSE Systems). All values were measured every 20 minutes and the average values for mice of the same genotypes at each time point are presented in a trend graph as mean \pm SEM. EE, $\mathrm{O}_{2}$ consumption, and $\mathrm{CO}_{2}$ 
production, and locomotor activity in the dark and light phases for both genotypes at $22^{\circ} \mathrm{C}$ and $30^{\circ} \mathrm{C}$ were determined by averaging the corresponding AUC for mice of the same genotypes.

Fecal energy content. Feces were collected for 3 days and stored at $-20^{\circ} \mathrm{C}$. The pooled feces were ovendried at $60^{\circ} \mathrm{C}$ for 30 hours. Dried feces $(0.8 \mathrm{~g})$ were used to determine the energy content by bomb calorimetry (IKA-Calorimeter C1, IKA-Werke GmbH\&Co. KG). Daily fecal energy loss (kJ) was calculated by multiplying fecal energy content $(\mathrm{kJ} / \mathrm{g})$ with daily fecal weight $(\mathrm{g})$.

Collection of fecal and cecal samples for SCFA analyses. Fecal pellets (3-6 per mouse) were freshly collected into headspace autosampler vials directly from the anus of mice on 3 different days within 2 weeks in the mid-dark phase. The last collection occurred at the time of sacrifice together with the cecal samples. Each sample was immediately weighed and stored at $-80^{\circ} \mathrm{C}$. The SCFA content of each sample was assessed using a TRACE GC Ultra gas chromatograph with a TriPlus RSH autosampler coupled to an ISQ quadrupole mass spectrometer (ThermoFisher Scientific).

Pellets were dissolved in $400 \mu \mathrm{l}$ phosphate buffer $(100 \mathrm{mM}$, pH 7.4) followed by the addition of $100 \mu 1$ internal standard (IS) solution containing a mixture of distilled water, $0.5 \mathrm{~g} / 1$ each of dissolved acetic acid$d_{4}$, propionic acid- $d_{5}$, and butyric acid- $d_{7}$ (Cambridge Isotope Laboratories), and $100 \mu 1$ of a $0.5 \mathrm{M} \mathrm{H}_{2} \mathrm{SO}_{4}$ solution. Vials were sealed and thoroughly vortexed until pellets were evenly suspended. The vials were directly subjected to headspace gas chromatography-mass spectrometry-based (HS-GC-MS-based) SCFA analysis. Identification was initially performed by comparing MS spectra and retention times with those of the external calibrators and against the NIST database. Quantification was performed using peak area to IS ratios against an external aqueous calibration (range 0.01-2.5 g/1 each) and evaluated using Quan Browser in Xcalibur 2.2 with the Genesis algorithm. Retention times and quantifier/qualifier ions for each compound were as follows (quantifiers underlined): acetic acid $7.81 \mathrm{~min}, m / z \underline{60}, 45$; propionic acid 10.2 $\min , m / z \underline{74}, 45$; butyric acid $13.2 \mathrm{~min}, m / z \underline{60}, 73$; acetic acid- $d_{4} 7.65 \mathrm{~min}, m / z \underline{63}, 46$; propionic acid- $d_{5}$ $9.93 \mathrm{~min}, \mathrm{~m} / z \underline{79}, 62$; butyric acid- $d_{7} 12.82 \mathrm{~min}, \mathrm{~m} / z \underline{63}$, 77. QC samples were analyzed repeatedly over the whole batch and were $\pm 20 \%$ of the spiked concentration. Final calculated concentrations were related to the applied feces wet weight.

Blood and urine biochemical measurements. Plasma levels of insulin (Ultra Sensitive ELISA Kit, Crystal Chem Inc.), NEFA (Zenbio, Inc.), and aldosterone (EIA-5298, DRG International Inc.) were determined using commercial kits according to the manufacturers' instructions. Plasma steroid hormones were measured using liquid chromatography-tandem mass spectrometry LC-MS/MS, as previously described (76). Plasma glucose, TG, cholesterol and creatinine levels were measured by the Zurich Integrative Rodent Physiology (ZIRP) core facility of the University of Zurich using standard techniques (UniCel DxC 800 Synchron Clinical System). Plasma and urinary electrolytes $\left(\mathrm{Na}^{+}, \mathrm{K}^{+}\right)$were determined using an EFOX 5053 flame photometer (Eppendorf) and urine creatinine was measured by the Jaffe method (77).

Liver TG content. Liver tissue (100-150 mg) was homogenized with $1 \mathrm{ml}$ of $5 \% \mathrm{NP}-40$, followed by twice heating to $90^{\circ} \mathrm{C}$ for 5 minutes and cooling down to room temperature. After centrifugation (13,000 $\mathrm{g}$ for 2 minutes) to remove insoluble material, the extracted TGs were diluted 10-fold with water and the amount was determined with a TG quantification kit (ab65336, Abacam). The concentration is expressed as $\mathrm{mg} / \mathrm{g}$ wet weight.

$q R T-P C R$. Total RNAs were extracted using the NucleoSpin RNA (Macherey-Nagel AG), followed by oligo(dT)-mediated reverse transcription using a RevertAid cDNA Synthesis Kit (ThermoFisher Scientific). The resultant cDNA was diluted 5-fold for qRT-PCR analysis with Master I SYBR Green qPCR Master Mix. qRT-PCR was performed in duplicate for each sample in a LightCycler 480 Real-Time PCR System according to the manufacturer's instructions (Roche). Relative amounts of mRNA were determined using the comparative $\mathrm{Ct}$ method and were normalized to GAPDH mRNA expression for which Ct values were not altered by genotypes or diets. The value of the control group was set to 100 . Primer sequences are listed in Supplemental Table 2. The gene expression array was performed using the $\mathrm{RT}^{2}$ Profiler PCR Array PAMM-049ZF following the manufacturer's protocol (Qiagen).

Citrate synthase activity assay. Perirenal fat $(60 \mathrm{mg})$ was ground to a fine powder in a liquid nitrogenprechilled mortar and pestle in $300 \mu \mathrm{l}$ sample buffer containing $100 \mathrm{mM}$ MOPs, $100 \mathrm{mM}$ bicin, and $3 \mathrm{mM}$ EDTA, pH 8.2. The tissue homogenate was thawed on ice and sonicated 3 times for 10 seconds each, separated by at least 30 seconds, to further disrupt the mitochondrial membranes. After centrifugation $\left(12,000 \mathrm{~g}, 7\right.$ minutes, $\left.4^{\circ} \mathrm{C}\right)$ of cell lysates to remove cell debris, the soluble fraction was harvested and its protein concentration was determined using the Bradford assay. 
Citrate synthase activity was assayed by measuring the oxaloacetate-dependent production of CoA-SH according to the method of Srere et al. (78). CoA-SH was made spectrophotometrically quantifiable by reacting it with DTNB to form TNB, which exhibits maximal absorbance at $412 \mathrm{~nm}$. Briefly, $10 \mu 1$ of sample containing $4 \mu \mathrm{g}$ protein was first mixed with $180 \mu \mathrm{l}$ assay solution containing $110 \mathrm{mM}$ MOPS, $110 \mathrm{mM}$

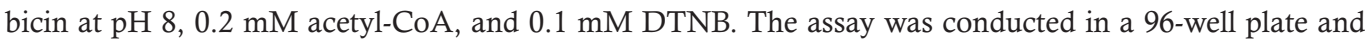
was started by adding $4 \mu 1$ of $10 \mathrm{mM}$ oxaloacetate. The plate was read at 1 -minute intervals for 30 minutes at $412 \mathrm{~nm}$. The absorbance values were plotted against time and the linear part of the curve was used to determine the enzyme activity. All samples were assayed in quadruplicate. Citrate synthase activities are reported as $\mathrm{U} / \mathrm{g}$ of protein.

Histology and immunofluorescence. Collected tissue slices roughly $3 \mathrm{~mm}$ thick were fixed with $10 \%$ formalin in PBS for 24 hours at $4^{\circ} \mathrm{C}$ and embedded in paraffin blocks. Ten-micrometer sections were deparaffinized and stained with $\mathrm{H} \& \mathrm{E}$. Images were acquired under a light microscope, and the size of adipocytes was determined using ImageJ software (NIH). For immunofluorescence, antigens of deparaffinized adipose sections were unmasked by pressure cooking with citric acid and trisodium citrate buffer before incubation with primary antibody at $4^{\circ} \mathrm{C}$ overnight, followed by secondary antibody incubation at room temperature for 1 hour (for sources and concentrations of antibodies used see Supplemental Figure 3). Images were acquired using a Leica DM 6000 fluorescence microscope.

Immunoblotting. Tissue lysates were prepared by homogenizing samples in $50 \mathrm{mM}$ HEPES, 150 $\mathrm{mM} \mathrm{NaCl}, 1 \mathrm{mM}$ EGTA, 10\% glycerol, 1\% Triton X-100, $50 \mathrm{mM} \mathrm{NaF}, 1.2 \mathrm{mg} / \mathrm{ml}$ NEM, $10 \mathrm{mM}$ Na-dipyrophosphate, $1 \mathrm{mM}$ DTT, and complete protease inhibitor cocktail and centrifuging (13,000 $g, 20$ minutes, $\left.4^{\circ} \mathrm{C}\right)$. Protein concentration was measured by the Bradford assay. Protein $(40 \mu \mathrm{g})$ was loaded on SDS-PAGE gels and transferred to a PVDF membrane (Immobilon, Millipore). After blocking for 1 hour in 5\% BSA or nonfat dry milk diluted in TBS containing 1\% Tween 20 (TBST), blots were incubated overnight with primary antibody at $4^{\circ} \mathrm{C}$. Following 3 washes with TBST, membranes were incubated with HRP-conjugated secondary antibody for 1 hour at room temperature (for sources and concentrations of antibodies used, see Supplemental Table 3). Proteins were detected using a chemiluminescence kit (GE Healthcare) and autoradiography films. Densitometric quantification was performed using ImageJ.

I.c.v. infusion. Ten- to 12 -week HFD-fed mice received chronic i.c.v. infusions of either aCSF (Harvard Apparatus) or aldosterone (Sigma-Aldrich, 9477) in aCSF (25 ng/h) for 4 weeks using osmotic minipumps (Alzet model 2004). The dose of aldosterone ( $25 \mathrm{ng} / \mathrm{h}$ ) used is supraphysiological and was based on a previous study (79).

The filled minipumps were connected to a brain cannula with a polycarbonate catheter (Brain Infusion Kit 2, Alzet) and primed in $37^{\circ} \mathrm{C}$ sterile $0.9 \%$ saline for 60 hours before surgery. Under $2 \%-3 \%$ isoflurane anesthesia, mice were fixed with the ear bars on a Model 940 stereotaxic frame (David Kopf Instruments), which provides $10-\mu \mathrm{m}$ resolution linear glass scales. A primed minipump was then positioned subcutaneously, and the brain cannula was unilaterally implanted into the lateral ventricle with the following coordinates: anterior-posterior -0.220 , medial-lateral +1.000 , dorsal-ventral -3.000 . The cannula was secured to the skull with liquid cyanoacrylate glue, and the skin was closed with 6-O absorbable suture. Standard aseptic surgery, prophylactic antibiotics, and analgesia procedures were applied. For all animals, the correct attachment of the cannula to the skull, and the patency of the cannula-minipump ensemble were confirmed at sacrifice.

Statistics. All data were analyzed using online GraphPad software (http://www.graphpad.com/quickcalcs/ ttest1/?Format=SD) or GraphPad Prism 7 and are presented as dot plots with mean \pm SEM unless otherwise indicated. Group differences were analyzed by unpaired 2-tailed Welch's $t$ test or Mann-Whitney $U$ test or 1-way ANOVA with Tukey's post hoc test when more than 2 groups were compared, depending on research hypothesis, and considered significant when $P<0.05$.

Study approval. All studies were approved by the Veterinary Office of the Canton of Zurich, Switzerland.

\section{Author contributions}

WHL conceived and designed the experiments in consultation with WL. WHL, CS, AES, GPL, AO, and NF performed the experiments. MH helped with the statistical analyses. WL provided resources and supervision. WHL drafted the manuscript. All authors edited the manuscript. 


\section{Acknowledgments}

We thank the ZIRP core facility for various biochemical measurements and Nino Jejelava, Myrtha Arnold, Beate Laube (ETH Zurich), Sebastien Desarzens, and Sandra Varum Tavares (University of Zurich) for the technical support in animal experiments, citrate synthase activity assay, and immunofluorescence. We thank Kim Zarse (ETH Zurich) and Steve Woods (University of Cincinnati) for valuable inputs. This work is supported by Swiss National Science Foundation (SNSF) Research grant 310030_153149 (to W. Langhans), SNSF Ambizione grant PZ00P3_142594/1; the National Centre of Competence in Research (NCCR) Kidney.CH Junior grant (to N. Faresse), NCCR kidney grant (to A. Odermatt); and the Institute of Evolutionary Medicine of the University of Zurich grant (to W.-H. Liao and W. Langhans) work of the Institute of Evolutionary Medicine is funded by Maxi Foundation.

Address correspondence to: Wan-Hui Liao or Wolfgang Langhans, Schorenstrasse 16, 8603 Schwerzenbach, Switzerland. Phone: 41.44.655.74.20; Email: whliao@ntu.edu.tw (W.-H. Liao); wolfgang-langhans@ethz.ch (W. Langhans).

1. Barrington EJ. The phylogeny of the endocrine system. Experientia. 1986;42(7):775-781.

2. Wagner CA. Effect of mineralocorticoids on acid-base balance. Nephron Physiol. 2014;128(1-2):26-34.

3. Brown NJ. Contribution of aldosterone to cardiovascular and renal inflammation and fibrosis. Nat Rev Nephrol. 2013;9(8):459-469.

4. Whaley-Connell A, Johnson MS, Sowers JR. Aldosterone: role in the cardiometabolic syndrome and resistant hypertension. Prog Cardiovasc Dis. 2010;52(5):401-409.

5. Navaneethan SD, Nigwekar SU, Sehgal AR, Strippoli GF. Aldosterone antagonists for preventing the progression of chronic kidney disease: a systematic review and meta-analysis. Clin J Am Soc Nephrol. 2009;4(3):542-551.

6. Maschio G, et al. Effect of the angiotensin-converting-enzyme inhibitor benazepril on the progression of chronic renal insufficiency. The Angiotensin-Converting-Enzyme Inhibition in Progressive Renal Insufficiency Study Group. N Engl J Med. 1996;334(15):939-945.

7. Lewis EF, et al. Impact of spironolactone on longitudinal changes in health-related quality of life in the treatment of preserved cardiac function heart failure with an aldosterone antagonist trial. Circ Heart Fail. 2016;9(3):e01937.

8. Zannad F, et al. Eplerenone in patients with systolic heart failure and mild symptoms. N Engl J Med. 2011;364(1):11-21.

9. Bomback AS, Klemmer PJ. The incidence and implications of aldosterone breakthrough. Nat Clin Pract Nephrol. 2007;3(9):486-492.

10. Luther JM, et al. Aldosterone decreases glucose-stimulated insulin secretion in vivo in mice and in murine islets. Diabetologia. 2011;54(8):2152-2163.

11. Bogman K, et al. Preclinical and early clinical profile of a highly selective and potent oral inhibitor of aldosterone synthase (CYP11B2). Hypertension. 2017;69(1):189-196.

12. Weldon SM, et al. Selectivity of BI 689648, a novel, highly selective aldosterone synthase inhibitor: Comparison with FAD286 and LCI699 in nonhuman primates. J Pharmacol Exp Ther. 2016;359(1):142-150.

13. Sloan-Lancaster J, Raddad E, Flynt A, Jin Y, Voelker J, Miller JW. LY3045697: Results from two randomized clinical trials of a novel inhibitor of aldosterone synthase. J Renin Angiotensin Aldosterone Syst. 2017;18(3):1470320317717883.

14. Hofmann A, et al. Aldosterone synthase inhibition improves glucose tolerance in Zucker diabetic fatty (ZDF) rats. Endocrinology. 2016;157(10):3844-3855.

15. Ménard J, et al. Aldosterone synthase inhibition: cardiorenal protection in animal disease models and translation of hormonal effects to human subjects. J Transl Med. 2014;12:340.

16. Mulder $\mathrm{P}$, et al. Aldosterone synthase inhibition improves cardiovascular function and structure in rats with heart failure: a comparison with spironolactone. Eur Heart J. 2008;29(17):2171-2179.

17. Lee $\mathrm{G}$, et al. Homeostatic responses in the adrenal cortex to the absence of aldosterone in mice. Endocrinology. 2005;146(6):2650-2656.

18. Goodfriend TL, Kelley DE, Goodpaster BH, Winters SJ. Visceral obesity and insulin resistance are associated with plasma aldosterone levels in women. Obes Res. 1999;7(4):355-362.

19. Makhanova N, et al. Kidney function in mice lacking aldosterone. Am J Physiol Renal Physiol. 2006;290(1):F61-F69.

20. Mitch WE. Metabolic and clinical consequences of metabolic acidosis. J Nephrol. 2006;19 Suppl 9:S70-S75

21. Bergman EN. Energy contributions of volatile fatty acids from the gastrointestinal tract in various species. Physiol Rev. 1990;70(2):567-590.

22. Jenkins HR, Fenton TR, McIntosh N, Dillon MJ, Milla PJ. Development of colonic sodium transport in early childhood and its regulation by aldosterone. Gut. 1990;31(2):194-197.

23. Jindrichová S, et al. Corticosteroid effect on Caco-2 cell lipids depends on cell differentiation. J Steroid Biochem Mol Biol. 2003;87(2-3):157-165

24. Wong JM, de Souza R, Kendall CW, Emam A, Jenkins DJ. Colonic health: fermentation and short chain fatty acids. J Clin Gastroenterol. 2006;40(3):235-243.

25. Milici-Emili G, Petit JM. Mechanical efficiency of breathing. J Appl Physiol. 1960;15:359-362.

26. Silberman H, Silberman AW. Parenteral nutrition, biochemistry and respiratory gas exchange. JPEN J Parenter Enteral Nutr. 1986;10(2):151-154

27. Gautier H, Bonora M, Trinh HC. Ventilatory and metabolic responses to cold and $\mathrm{CO}_{2}$ in intact and carotid body-denervated awake rats. J Appl Physiol. 1993;75(6):2570-2579. 
28. Guerra C, Koza RA, Yamashita H, Walsh K, Kozak LP. Emergence of brown adipocytes in white fat in mice is under genetic control. Effects on body weight and adiposity. J Clin Invest. 1998;102(2):412-420

29. Larsen S, et al. Biomarkers of mitochondrial content in skeletal muscle of healthy young human subjects. J Physiol (Lond). 2012;590(14):3349-3360.

30. Bogacka I, Xie H, Bray GA, Smith SR. Pioglitazone induces mitochondrial biogenesis in human subcutaneous adipose tissue in vivo. Diabetes. 2005;54(5):1392-1399.

31. Giordano A, et al. Regional-dependent increase of sympathetic innervation in rat white adipose tissue during prolonged fasting J Histochem Cytochem. 2005;53(6):679-687.

32. Jéquier E. Energy expenditure in obesity. Clin Endocrinol Metab. 1984;13(3):563-580.

33. Cooling J, Blundell J. Differences in energy expenditure and substrate oxidation between habitual high fat and low fat consumers (phenotypes). Int J Obes Relat Metab Disord. 1998;22(7):612-618.

34. Zhang YaM, Ai-Qun Gong, Min Lu, Qun Lu, Min Tian, Gang. Red blood cell level is increased in obese but not in non-obese patients with coronary heart disease. J Geriatr Cardiol. 2010;7(3-4):143-146.

35. Samocha-Bonet D, et al. Platelet counts and platelet activation markers in obese subjects. Mediators Inflamm. 2008;2008:834153.

36. Dixon JB, O'Brien PE. Obesity and the white blood cell count: changes with sustained weight loss. Obes Surg. 2006;16(3):251-257.

37. Rajan S, et al. Chronic hyperinsulinemia reduces insulin sensitivity and metabolic functions of brown adipocyte. $J$ Endocrinol. 2016;230(3):275-290.

38. Mehran AE, et al. Hyperinsulinemia drives diet-induced obesity independently of brain insulin production. Cell Metab. 2012;16(6):723-737.

39. Hsu IR, Kim SP, Kabir M, Bergman RN. Metabolic syndrome, hyperinsulinemia, and cancer. Am J Clin Nutr. 2007;86(3):s867-s871.

40. Cannon B, Nedergaard J. Brown adipose tissue: function and physiological significance. Physiol Rev. 2004;84(1):277-359.

41. Gomez-Sanchez EP, Ahmad N, Romero DG, Gomez-Sanchez CE. Is aldosterone synthesized within the rat brain? Am JPhysiol Endocrinol Metab. 2005;288(2):E342-E346.

42. Chang HC, et al. Resveratrol attenuates high-fat diet-induced disruption of the blood-brain barrier and protects brain neurons from apoptotic insults. J Agric Food Chem. 2014;62(15):3466-3475

43. Saeed AA, et al. Effects of a disrupted blood-brain barrier on cholesterol homeostasis in the brain. J Biol Chem. 2014;289(34):23712-23722.

44. Weisinger RS, Woods SC. Aldosterone-elicited sodium appetite. Endocrinology. 1971;89(2):538-544.

45. Portrat-Doyen S, et al. Isolated aldosterone synthase deficiency caused by simultaneous E198D and V386A mutations in the CYP11B2 gene. J Clin Endocrinol Metab. 1998;83(11):4156-4161.

46. Hui E, et al. The clinical significance of aldosterone synthase deficiency: report of a novel mutation in the CYP11B2 gene. BMC Endocr Disord. 2014;14:29.

47. Jessen CL, Christensen JH, Birkebaek NH, Rittig S. Homozygosity for a mutation in the CYP11B2 gene in an infant with congenital corticosterone methyl oxidase deficiency type II. Acta Paediatr. 2012;101(11):e519-e525.

48. Challa A, Krieg RJ, Thabet MA, Veldhuis JD, Chan JC. Metabolic acidosis inhibits growth hormone secretion in rats: mechanism of growth retardation. Am J Physiol. 1993;265(4 Pt 1):E547-E553.

49. Brüngger M, Hulter HN, Krapf R. Effect of chronic metabolic acidosis on the growth hormone/IGF-1 endocrine axis: new cause of growth hormone insensitivity in humans. Kidney Int. 1997;51(1):216-221.

50. Green J, Maor G. Effect of metabolic acidosis on the growth hormone/IGF-I endocrine axis in skeletal growth centers. Kidney Int. 2000;57(6):2258-2267.

51. Nash MA, Torrado AD, Greifer I, Spitzer A, Edelmann CM. Renal tubular acidosis in infants and children. Clinical course, response to treatment, and prognosis. J Pediatr. 1972;80(5):738-748.

52. McSherry E, Morris RC. Attainment and maintenance of normal stature with alkali therapy in infants and children with classic renal tubular acidosis. J Clin Invest. 1978;61(2):509-527.

53. Saupe KW, Smith CA, Henderson KS, Dempsey JA. Respiratory muscle recruitment during selective central and peripheral chemoreceptor stimulation in awake dogs. J Physiol (Lond). 1992;448:613-631.

54. Romagnoli I, et al. Chest wall kinematics and respiratory muscle coordinated action during hypercapnia in healthy males. Eur $J$ Appl Physiol. 2004;91(5-6):525-533.

55. Cai B, et al. Effect of supplementing a high-fat, low-carbohydrate enteral formula in COPD patients. Nutrition. $2003 ; 19(3): 229-232$

56. al-Saady NM, Blackmore CM, Bennett ED. High fat, low carbohydrate, enteral feeding lowers $\mathrm{PaCO}_{2}$ and reduces the period of ventilation in artificially ventilated patients. Intensive Care Med. 1989;15(5):290-295.

57. Kuo CD, Shiao GM, Lee JD. The effects of high-fat and high-carbohydrate diet loads on gas exchange and ventilation in COPD patients and normal subjects. Chest. 1993;104(1):189-196.

58. Hsieh MJ, Yang TM, Tsai YH. Nutritional supplementation in patients with chronic obstructive pulmonary disease. J Formos Med Assoc. 2016;115(8):595-601.

59. Efthimiou J, Mounsey PJ, Benson DN, Madgwick R, Coles SJ, Benson MK. Effect of carbohydrate rich versus fat rich loads on gas exchange and walking performance in patients with chronic obstructive lung disease. Thorax. 1992;47(6):451-456.

60. Noakes M, Foster PR, Keogh JB, James AP, Mamo JC, Clifton PM. Comparison of isocaloric very low carbohydrate/high saturated fat and high carbohydrate/low saturated fat diets on body composition and cardiovascular risk. Nutr Metab (Lond). 2006;3:7.

61. Cruzen C, Colman RJ. Effects of caloric restriction on cardiovascular aging in non-human primates and humans. Clin Geriatr Med. 2009;25(4):733-743.

62. Narkiewicz K, Pesek CA, van de Borne PJ, Kato M, Somers VK. Enhanced sympathetic and ventilatory responses to central chemoreflex activation in heart failure. Circulation. 1999;100(3):262-267.

63. Narkiewicz K, Kato M, Pesek CA, Somers VK. Human obesity is characterized by a selective potentiation of central chemoreflex sensitivity. Hypertension. 1999;33(5):1153-1158

64. Saltiel AR, Kahn CR. Insulin signalling and the regulation of glucose and lipid metabolism. Nature. 2001;414(6865):799-806. 
65. Klarr SA, Keep RF, Betz AL. Chronic central potassium infusion prevents deoxycorticosterone-salt hypertension in rats. Am J Physiol. 1995;268(2 Pt 2):H646-H652.

66. Atarashi K, et al. Effects of intracerebroventricular infusion of aldosterone on blood pressure and sodium and potassium concentrations in cerebral spinal fluid in rats. Clin Exp Hypertens A. 1988;10 Suppl 1:317-322.

67. Guo C, et al. Mineralocorticoid receptor blockade reverses obesity-related changes in expression of adiponectin, peroxisome proliferator-activated receptor-gamma, and proinflammatory adipokines. Circulation. 2008;117(17):2253-2261.

68. Armani A, et al. Mineralocorticoid receptor antagonism induces browning of white adipose tissue through impairment of autophagy and prevents adipocyte dysfunction in high-fat-diet-fed mice. FASEB J. 2014;28(8):3745-3757.

69. Funder JW. Mineralocorticoid receptor antagonists: emerging roles in cardiovascular medicine. Integr Blood Press Control. 2013;6:129-138.

70. Hervey E, Hervey GR. The effects of progesterone on body weight and composition in the rat. J Endocrinol. 1967;37(4):361-381

71. Marzolla V, et al. The role of the mineralocorticoid receptor in adipocyte biology and fat metabolism. Mol Cell Endocrinol. 2012;350(2):281-288.

72. de Kloet ER, et al. Brain mineralocorticoid receptors and centrally regulated functions. Kidney Int. 2000;57(4):1329-1336.

73. Luther JM, et al. Aldosterone deficiency and mineralocorticoid receptor antagonism prevent angiotensin II-induced cardiac, renal, and vascular injury. Kidney Int. 2012;82(6):643-651.

74. Kanarek-Kucner J, et al. Insufficiency of the zona glomerulosa of the adrenal cortex and progressive kidney insufficiency following unilateral adrenalectomy - case report and discussion. Blood Press. 2018:1-9.

75. Krege JH, Hodgin JB, Hagaman JR, Smithies O. A noninvasive computerized tail-cuff system for measuring blood pressure in mice. Hypertension. 1995;25(5):1111-1115.

76. Strajhar P, et al. Acute effects of lysergic acid diethylamide on circulating steroid levels in healthy subjects. J Neuroendocrinol. 2016;28(3):12374

77. Seaton B, Ali A. Simplified manual high performance clinical chemistry methods for developing countries. Med Lab Sci. 1984;41(4):327-336

78. Srere P. [1] Citrate synthase:[EC 4.1. 3.7. Citrate oxaloacetate-lyase (CoA-acetylating)]. Methods Enzymol. 1969;13:3-11.

79. Koneru B, Bathina CS, Cherry BH, Mifflin SW. Mineralocorticoid receptor in the NTS stimulates saline intake during fourth ventricular infusions of aldosterone. Am J Physiol Regul Integr Comp Physiol. 2014;306(1):R61-R66. 